\title{
Multivariate portmanteau test for structural VARMA models with uncorrelated but non-independent error terms
}

\author{
Y. Boubacar Mainassara ${ }^{a}$ \\ ${ }^{a}$ Université Lille III, EQUIPPE, BP 60 149, 59653 Villeneuve d'Ascq cedex, France.
}

\begin{abstract}
We consider portmanteau tests for testing the adequacy of structural vector autoregressive moving-average (VARMA) models under the assumption that the errors are uncorrelated but not necessarily independent. The structural forms are mainly used in econometrics to introduce instantaneous relationships between economic variables. We first study the joint distribution of the quasi-maximum likelihood estimator (QMLE) and the noise empirical autocovariances. We then derive the asymptotic distribution of residual empirical autocovariances and autocorrelations under weak assumptions on the noise. We deduce the asymptotic distribution of the Ljung-Box (or Box-Pierce) portmanteau statistics in this framework. It is shown that the asymptotic distribution of the portmanteau tests is that of a weighted sum of independent chi-squared random variables, which can be quite different from the usual chisquared approximation used under independent and identically distributed (iid) assumptions on the noise. Hence we propose a method to adjust the critical values of the portmanteau tests. Monte carlo experiments illustrate the finite sample performance of the modified portmanteau test.
\end{abstract}

Keywords: Goodness-of-fit test, QMLE/LSE, Box-Pierce and Ljung-Box portmanteau tests, Residual autocorrelation, Structural representation, Weak VARMA models.

Email address: mailto: yacouba.babacarmainassara@etu.univ-lille3.fr (Y. Boubacar Mainassara)

URL: http://perso.univ-lille3.fr/ yboubacarmai/(Y. Boubacar Mainassara) 


\section{Introduction}

Consider a $d$-dimensional stationary process $\left(X_{t}\right)$ satisfying a $\operatorname{VARMA}(p, q)$ representation of the form

$$
A_{00} X_{t}-\sum_{i=1}^{p} A_{0 i} X_{t-i}=B_{00} \epsilon_{t}-\sum_{i=1}^{q} B_{0 i} \epsilon_{t-i}, \quad \forall t \in \mathbb{Z}=\{0, \pm 1, \ldots\} .
$$

When $A_{00}=B_{00}=I_{d}$, the $\operatorname{VARMA}(p, q)$ representation is said to be in reduced form. Otherwise, it is said to be structural. The structural forms are mainly used in econometrics to introduce instantaneous relationships between economic variables. The representation (1) is said to satisfy a weak $\operatorname{VARMA}(p, q)$ representation if $\epsilon_{t}$ is a weak white noise, namely a stationary sequence of centered and uncorrelated random variables with a non singular variance $\Sigma_{0}$. It is customary to say that $\left(X_{t}\right)$ is a strong $\operatorname{VARMA}(p, q)$ model if $\left(\epsilon_{t}\right)$ is an iid sequence of random variables with mean 0 and common variance matrix $\Sigma_{0}$ (i.e. strong white noise). A strong white noise is obviously a weak white noise, because independence entails uncorrelatedness, but the reverse is not true. Between weak and strong $\operatorname{VARMA}(p, q)$ representations, one can say that $(1)$ is a semi-strong $\operatorname{VARMA}(p, q)$ representation if $\left(\epsilon_{t}\right)$ is a stationary martingale difference (i.e. semi-strong white noise).

The structural $\operatorname{VARMA}(p, q)$ representation (1) can be rewritten in a standard reduced $\operatorname{VARMA}(p, q)$ form if the matrices $A_{00}$ and $B_{00}$ are non singular. Indeed, premultiplying (1) by $A_{00}^{-1}$ and introducing the innovation process $e_{t}=A_{00}^{-1} B_{00} \epsilon_{t}$, with non singular variance $\Sigma_{e 0}=A_{00}^{-1} B_{00} \Sigma_{0} B_{00}^{\prime} A_{00}^{-1^{\prime}}$, we obtain the reduced VARMA representation

$$
X_{t}-\sum_{i=1}^{p} A_{00}^{-1} A_{0 i} X_{t-i}=e_{t}-\sum_{i=1}^{q} A_{00}^{-1} B_{0 i} B_{00}^{-1} A_{00} e_{t-i}
$$

The structural form (1) allows to handle seasonal models, instantaneous economic relationships, VARMA in the so-called echelon form representation, and many other constrained VARMA representations (see Lütkepohl, 2005, chap. 12). The reduced form (2) is more practical from a statistical viewpoint, because it gives the forecasts of each component of $\left(X_{t}\right)$ according to the past values of the set of the components.

The above discussion shows that VARMA representations are not unique, that is, a given process $\left(X_{t}\right)$ can be written in reduced form or in structural 
form by premultiplying by any non singular $(d \times d)$ matrix. Of course, in order to ensure the uniqueness of a VARMA representation, constraints are necessary for the identifiability of the $(p+q+3) d^{2}$ elements of the matrices involved in the VARMA equation (1). In contrast, the echelon form guarantees uniqueness of the VARMA representation (see also Lütkepohl, 2005). The echelon form is the most widely identified VARMA representation employed in the literature. The identifiability of VARMA processes has been studied in particular by Hannan (1976) who gave several procedures ensuring identifiability.

The validity of the different steps of the traditional methodology of Box and Jenkins, identification, estimation and validation, depends on the noise properties. After identification and estimation of the VARMA processes, the next important step in the VARMA modeling consists in checking if the estimated model fits satisfactorily the data. This adequacy checking step allows to validate or invalidate the choice of the orders $p$ and $q$. In $\operatorname{VARMA}(p, q)$ models, the choice of $p$ and $q$ is particularly important because the number of parameters, $(p+q+2) d^{2}$, quickly increases with $p$ and $q$, which entails statistical difficulties.

In particular, the selection of too large orders $p$ and $q$ has the effect of introducing terms that are not necessarily relevant in the model. Overidentification thus generally leads to a loss of precision in parameter estimation. Conversely, the selection of too small orders $p$ and $q$ causes loss of some information, that results in a lack of asymptotic precision for the predictions.

Thus it is important to check the validity of a $\operatorname{VARMA}(p, q)$ model, for given orders $p$ and $q$. This paper is devoted to the problem of the validation step of VARMA representations of multivariate processes. Based on the residual empirical autocorrelations, Box and Pierce (1970) (BP hereafter) derived a goodness-of-fit test, the portmanteau test, for univariate strong ARMA models. Ljung and Box (1978) (LB hereafter) proposed a modified portmanteau test which is nowadays one of the most popular diagnostic checking tool in ARMA modeling of time series. The multivariate version of the BP portmanteau statistic was introduced by Chitturi (1974). We use the portmanteau tests considered by Chitturi (1974) and Hosking (1980) for checking the overall significance of the residual autocorrelations of a $\operatorname{VARMA}(p, q)$ model (see also Chitturi (1976), Hosking (1981a,b), Li and McLeod (1981), Ahn (1988). Hosking (1981a) gave several equivalent forms of this statistic. Arbués (2008) proposed an extended portmanteau test for VARMA models with mixing nonlinear constraints. 
The works on the multivariate version of the portmanteau statistic are generally performed under the assumption that the errors $\epsilon_{t}$ are independent. This independence assumption is often considered too restrictive by practitioners. It precludes conditional heteroscedasticity and/or other forms of nonlinearity (see Francq and Zakoïan, 2005, for a review on weak univariate ARMA models). Relaxing this independence assumption allows to cover linear representations of general nonlinear processes and to extend the range of application of the VARMA models.

The asymptotic theory of weak ARMA model validation is mainly limited to the univariate framework (see Francq, Roy and Zakoïan, 2005, hereafter FRZ). In the multivariate analysis, a notable exception is Francq and Raïssi (2007) who study portmanteau tests for weak VAR models. We will generalize this result to VARMA models. This extension raises difficult problems. First, non trivial constraints on the parameters must be imposed for identifiability of the parameters (see Reinsel, 1997, Lütkepohl, 2005). Secondly, the implementation of standard estimation methods (for instance the Gaussian quasi-maximum likelihood estimation) is not obvious because this requires a constrained high-dimensional optimization (see also Lütkepohl, 2005). These technical difficulties certainly explain why VAR models are much more used than VARMA in applied works.

Recently Boubacar Mainassara and Francq (2010) (hereafter BMF) study the consistency and the asymptotic normality of the QMLE for a weak VARMA model. The QMLE is obtained by maximizing a function that would be the logarithm of the likelihood function if the process was Gaussian, but is not equal to it when the process $\left(\epsilon_{t}\right)$ is not iid Gaussian. The function that is maximized is often called quasi-likelihood. The QMLE can also be viewed as a nonlinear least squared estimator (LSE). Dufour and Pelletier (2005) and Boubacar Mainassara (in a working paper, 2010) study the choice of the orders $p$ and $q$ of weak VARMA models using information criteria, Chabot-Hallé and Duchesne (2008) study the asymptotic distribution of LSE and portmanteau test for semi-strong VAR. The main goal of the present paper is to complete the available results concerning the statistical analysis of weak VARMA models by considering the adequacy problem under general error terms. We proceed to study the behaviour of the goodness-of fit portmanteau tests when the $\epsilon_{t}$ are not independent. It is shown that the standard portmanteau tests can be quite misleading in the framework of non independent errors. Consequently, a modified version of these tests is proposed. 
The paper is organized as follows. Section 2 presents the parametrization and assumptions used in the sequel. In Section 3, we recall the results on the QMLE asymptotic distribution obtained by BMF when $\left(\epsilon_{t}\right)$ satisfies mild mixing assumptions. Section 4 is devoted to the derivation of the joint distribution of the QMLE and the noise empirical autocovariances. In Section 5, we derive the asymptotic distribution of residual empirical autocovariances and autocorrelations under weak assumptions on the noise. In Section 6, it is shown how the standard Ljung-Box (or Box-Pierce) portmanteau tests must be adapted in the case of VARMA models with nonindependent innovations. Numerical experiments are presented in Section 7 and we provide a conclusion in Section 8. The proofs of the main results are collected in the appendix.

\section{Parametrization and assumptions}

Let $\left[A_{00} \ldots A_{0 p} B_{00} \ldots B_{0 q}\right]$ be the $d \times(p+q+2) d$ matrix of VAR and MA coefficients involved in the VARMA equation (1). The matrix $\Sigma_{0}$ is considered as a nuisance parameter. The parameter of interest is denoted $\theta_{0}$, where $\theta_{0}$ belongs to the parameter space $\Theta \subset \mathbb{R}^{k_{0}}$, and $k_{0}$ is the number of unknown parameters, which is typically much smaller that $(p+q+3) d^{2}$. The matrices $A_{00}, \ldots A_{0 p}, B_{00}, \ldots B_{0 q}$ involved in (1) and $\Sigma_{0}$ are specified by $\theta_{0}$. More precisely, we write $A_{0 i}=A_{i}\left(\theta_{0}\right)$ and $B_{0 j}=B_{j}\left(\theta_{0}\right)$ for $i=0, \ldots, p$ and $j=0, \ldots, q$, and $\Sigma_{0}=\Sigma\left(\theta_{0}\right)$. To ensure the consistence and the asymptotic normality of the QMLE, we assume that the parametrization satisfies the following smoothness conditions.

A1: $\quad$ The functions $\theta \mapsto A_{i}(\theta) i=0, \ldots, p, \theta \mapsto B_{j}(\theta) j=0, \ldots, q$ and $\theta \mapsto \Sigma(\theta)$ admit continuous third order derivatives for all $\theta \in \Theta$.

For simplicity, we now write $A_{i}, B_{j}$ and $\Sigma$ instead of $A_{i}(\theta), B_{j}(\theta)$ and $\Sigma(\theta)$. Let $A_{\theta}(z)=A_{0}-\sum_{i=1}^{p} A_{i} z^{i}$ and $B_{\theta}(z)=B_{0}-\sum_{i=1}^{q} B_{i} z^{i}$. We assume that $\Theta$ corresponds to stable and invertible representations, namely

A2: $\quad$ for all $\theta \in \Theta$, we have $\operatorname{det} A_{\theta}(z) \operatorname{det} B_{\theta}(z) \neq 0$ for all $|z| \leq 1$.

To ensure the strong consistency of the QMLE, a compactness assumption is required.

A3: We have $\theta_{0} \in \Theta$, where $\Theta$ is compact. 
The structural VARMA model (1) can be written more compactly as

$A_{\theta_{0}}(L) X_{t}=B_{\theta_{0}}(L) \epsilon_{t}$ where $A_{\theta_{0}}(L)=A_{00}-\sum_{i=1}^{p} A_{0 i} L^{i}, B_{\theta_{0}}(L)=B_{00}-\sum_{i=1}^{q} B_{0 i} L^{i}$

and where $L$ is the backward operator.

A4: The process $\left(\epsilon_{t}\right)$ is stationary and ergodic.

Note that A4 is entailed by the uncorrelated innovations, but not by the iid innovations. In view of $(3), X_{t}=A_{\theta_{0}}^{-1}(L) B_{\theta_{0}}(L) \epsilon_{t}$ and $\epsilon_{t}=B_{\theta_{0}}^{-1}(L) A_{\theta_{0}}(L) X_{t}$, $\left(\epsilon_{t}\right)$ can be replaced by $\left(X_{t}\right)$ in $\mathbf{A} 4$. In the structural VARMA model (1), the assumption $\mathbf{A} \mathbf{2}$ does not guarantee the identifiability of the parameter. Thus, we make the following global assumption for all $\theta \in \Theta$.

A5: For all $\theta \in \Theta$ such that $\theta \neq \theta_{0}$, either the transfer functions $A_{0}^{-1} B_{0} B_{\theta}^{-1}(z) A_{\theta}(z) \neq A_{00}^{-1} B_{00} B_{\theta_{0}}^{-1}(z) A_{\theta_{0}}(z)$ for some $z \in \mathbb{C}$, or $A_{0}^{-1} B_{0} \Sigma B_{0}^{\prime} A_{0}^{-1^{\prime}} \neq A_{00}^{-1} B_{00} \Sigma_{0} B_{00}^{\prime} A_{00}^{-1^{\prime}}$.

In the reduced VARMA representation (2), note that, the last condition in A5 can be dropped, but may be important for structural VARMA forms. In particular, A5 is satisfied when we impose: $A_{0}=B_{0}=\mathrm{I}_{d}, \mathbf{A} \mathbf{2}$, the common left divisors of $A_{\theta}(L)$ and $B_{\theta}(L)$ are unimodular (i.e. with nonzero constant determinant), and the matrix $\left[A_{p}: B_{q}\right]$ is of full rank. For the asymptotic normality of the QMLE, additional assumptions are required. It is necessary to assume that $\theta_{0}$ is not on the boundary of the parameter space $\Theta$.

A6: $\quad$ We have $\theta_{0} \in \stackrel{\circ}{\Theta}$, where $\stackrel{\circ}{\Theta}$ denotes the interior of $\Theta$.

We now introduce, as in Francq and Zakoïan (1998) (hereafter FZ) the strong mixing coefficients of a stationary process $Z=\left(Z_{t}\right)$ denoted by

$$
\alpha_{Z}(h)=\sup _{A \in \sigma\left(Z_{u}, u \leq t\right), B \in \sigma\left(X_{u}, u \geq t+h\right)}|P(A \cap B)-P(A) P(B)|,
$$

measuring the temporal dependence of the process $Z$. Denoting by $\|Z\|$ the Euclidean norm of $Z$.

A7: $\quad$ We have $E\left\|\epsilon_{t}\right\|^{4+2 \nu}<\infty$ and $\sum_{k=0}^{\infty}\left\{\alpha_{\epsilon}(k)\right\}^{\frac{\nu}{2+\nu}}<\infty$ for some $\nu>0$.

Note that assumption A7 does not require independence of the noise, nor the fact that it is a martingale difference. 


\section{Quasi-maximum likelihood estimation}

For all $\theta \in \Theta$, let $A_{0}=A_{0}(\theta), \ldots, A_{p}=A_{p}(\theta), B_{0}=B_{0}(\theta), \ldots, B_{q}=$ $B_{q}(\theta)$ and $\Sigma=\Sigma(\theta)$. Note that from $\mathbf{A 2}$, the matrices $A_{0}$ and $B_{0}$ are invertible. Thus, the structural representation (1) can be rewritten as the reduced VARMA representation (2). For the sake of simplicity, we omit the notation $\theta$ in all quantities taken at the true value $\theta_{0}$. For all $\theta \in \Theta$, the assumption on the MA polynomial (from A2) implies that there exists a sequence of constants matrices $\left(C_{i}(\theta)\right)$ such that $\sum_{i=1}^{\infty}\left\|C_{i}(\theta)\right\|<\infty$ and

$$
e_{t}(\theta)=X_{t}-\sum_{i=1}^{\infty} C_{i}(\theta) X_{t-i} .
$$

Given a realization $X_{1}, X_{2}, \ldots, X_{n}$ satisfying the VARMA representation (1), the variable $e_{t}(\theta)$ can be approximated, for $0<t \leq n$, by $\tilde{e}_{t}(\theta)$ defined recursively by

$$
\tilde{e}_{t}(\theta)=X_{t}-\sum_{i=1}^{p} A_{0}^{-1} A_{i} X_{t-i}+\sum_{i=1}^{q} A_{0}^{-1} B_{i} B_{0}^{-1} A_{0} \tilde{e}_{t-i}(\theta),
$$

where the unknown initial values are set to zero: $\tilde{e}_{0}(\theta)=\cdots=\tilde{e}_{1-q}(\theta)=$ $X_{0}=\cdots=X_{1-p}=0$. The Gaussian quasi-likelihood is given by

$$
\tilde{\mathrm{L}}_{n}\left(\theta, \Sigma_{e}\right)=\prod_{t=1}^{n} \frac{1}{(2 \pi)^{d / 2} \sqrt{\operatorname{det} \Sigma_{e}}} \exp \left\{-\frac{1}{2} \tilde{e}_{t}^{\prime}(\theta) \Sigma_{e}^{-1} \tilde{e}_{t}(\theta)\right\}, \quad \Sigma_{e}=A_{0}^{-1} B_{0} \Sigma B_{0}^{\prime} A_{0}^{-1^{\prime}} .
$$

A QMLE of $\left(\theta, \Sigma_{e}\right)$ is a measurable solution $\left(\hat{\theta}_{n}, \hat{\Sigma}_{e}\right)$ of

$$
\left(\hat{\theta}_{n}, \hat{\Sigma}_{e}\right)=\arg \min _{\theta, \Sigma_{e}}\left\{\log \left(\operatorname{det} \Sigma_{e}\right)+\frac{1}{n} \sum_{t=1}^{n} \tilde{e}_{t}(\theta) \Sigma_{e}^{-1} \tilde{e}_{t}^{\prime}(\theta)\right\} .
$$

We use the matrix $M_{\theta_{0}}$ of the coefficients of the reduced form (2), where

$$
M_{\theta_{0}}=\left[A_{00}^{-1} A_{01}: \cdots: A_{00}^{-1} A_{0 p}: A_{00}^{-1} B_{01} B_{00}^{-1} A_{00}: \cdots: A_{00}^{-1} B_{0 q} B_{00}^{-1} A_{00}\right] .
$$

Now, we need a local identifiability assumption which completes A5 and specifies how this matrix depends on the parameter $\theta_{0}$. We denote by $A \otimes B$ the Kronecker product of two matrices $A$ and $B$, and by $\operatorname{vec}(A)$ the vector obtained by stacking the columns of $A$. Let $\dot{M}_{\theta_{0}}$ be the matrix $\partial \operatorname{vec}\left(M_{\theta}\right) / \partial \theta^{\prime}$ evaluated at $\theta_{0}$. 
A8: $\quad$ The matrix $\dot{M}_{\theta_{0}}$ is of full rank $k_{0}$.

Under the following additional assumption, BMF showed the consistency and the asymptotic normality of the QMLE of a weak VARMA model (see Theorem 1 in BMF). One of the most popular estimation procedures is that of the least squares estimation. For the processes of the form (2), under A1A8, it can be shown (see e.g. Theorem 2 in BMF), that the LSE of $\theta$ coincides with the QMLE. Then under the assumptions A1-A8, BMF showed that $\hat{\theta}_{n} \rightarrow \theta_{0}$ a.s. as $n \rightarrow \infty$ and $\sqrt{n}\left(\hat{\theta}_{n}-\theta_{0}\right)$ is asymptotically normal with mean 0 and covariance matrix $\Sigma_{\hat{\theta}_{n}}:=J^{-1} I J^{-1}$, where $J=J\left(\theta_{0}, \Sigma_{e 0}\right)$ and $I=I\left(\theta_{0}, \Sigma_{e 0}\right)$, with

$$
J\left(\theta, \Sigma_{e}\right)=\lim _{n \rightarrow \infty} \frac{-2}{n} \frac{\partial^{2}}{\partial \theta \partial \theta^{\prime}} \log \tilde{\mathrm{L}}_{n}\left(\theta, \Sigma_{e}\right) \quad \text { a.s. }
$$

and

$$
I\left(\theta, \Sigma_{e}\right)=\lim _{n \rightarrow \infty} \operatorname{Var} \frac{2}{\sqrt{n}} \frac{\partial}{\partial \theta} \log \tilde{\mathrm{L}}_{n}\left(\theta, \Sigma_{e}\right) .
$$

In the standard strong VARMA case, i.e. when A4 is replaced by the assumption that $\left(\epsilon_{t}\right)$ is an iid sequence, we have $I=2 J$, so that $\Sigma_{\hat{\theta}_{n}}=2 J^{-1}$.

\section{Joint distribution of $\hat{\theta}_{\boldsymbol{n}}$ and the noise empirical autocovariances}

Let $\hat{e}_{t}=\tilde{e}_{t}\left(\hat{\theta}_{n}\right)$ be the quasi-maximum likelihood residuals when $p>0$ or $q>0$, and let $\hat{e}_{t}=e_{t}=X_{t}$ when $p=q=0$. When $p+q \neq 0$, we have $\hat{e}_{t}=0$ for $t \leq 0$ and $t>n$ and

$$
\hat{e}_{t}=X_{t}-\sum_{i=1}^{p} A_{0}^{-1}\left(\hat{\theta}_{n}\right) A_{i}\left(\hat{\theta}_{n}\right) \hat{X}_{t-i}+\sum_{i=1}^{q} A_{0}^{-1}\left(\hat{\theta}_{n}\right) B_{i}\left(\hat{\theta}_{n}\right) B_{0}^{-1}\left(\hat{\theta}_{n}\right) A_{0}\left(\hat{\theta}_{n}\right) \hat{e}_{t-i},
$$

for $t=1, \ldots, n$, with $\hat{X}_{t}=0$ for $t \leq 0$ and $\hat{X}_{t}=X_{t}$ for $t \geq 1$. We denote by

$$
\gamma(h)=\frac{1}{n} \sum_{t=h+1}^{n} e_{t} e_{t-h}^{\prime} \quad \text { and } \quad \hat{\Gamma}_{e}(h)=\frac{1}{n} \sum_{t=h+1}^{n} \hat{e}_{t} \hat{e}_{t-h}^{\prime}
$$

the white noise "empirical" autocovariances and residual autocovariances. It should be noted that $\gamma(h)$ is not a statistic (unless if $p=q=0$ ) because it depends on the unobserved innovations $e_{t}$. For a fixed integer $m \geq 1$, let

$\gamma_{m}=\left(\{\operatorname{vec} \gamma(1)\}^{\prime}, \ldots,\{\operatorname{vec} \gamma(m)\}^{\prime}\right)^{\prime}, \hat{\Gamma}_{m}=\left(\left\{\operatorname{vec} \hat{\Gamma}_{e}(1)\right\}^{\prime}, \ldots,\left\{\operatorname{vec} \hat{\Gamma}_{e}(m)\right\}^{\prime}\right)^{\prime}$ 
and

$$
\Gamma\left(\ell, \ell^{\prime}\right)=\sum_{h=-\infty}^{\infty} E\left(\left\{e_{t-\ell} \otimes e_{t}\right\}\left\{e_{t-h-\ell^{\prime}} \otimes e_{t-h}\right\}^{\prime}\right), \quad \text { for } \quad\left(\ell, \ell^{\prime}\right) \neq(0,0) .
$$

For the univariate ARMA model, FRZ have showed that $\sum_{h=-\infty}^{\infty}\left|E e_{t} e_{t+\ell} e_{t+h} e_{t+h+\ell^{\prime}}\right|<+\infty$ (see Lemma A.1), which in turn implies the existence of $\Gamma\left(\ell, \ell^{\prime}\right)$. We can generalize this result for the VARMA models. Then we obtain $\sum_{h=-\infty}^{\infty}\left\|E\left\{e_{t-\ell} \otimes e_{t}\right\}\left\{e_{t-h-\ell^{\prime}} \otimes e_{t-h}\right\}^{\prime}\right\|<+\infty$. The proof is similar to the univariate case.

We are now able to state the following Theorem, which is an extension of a result given in FRZ.

Theorem 4.1. Assume that $p>0$ or $q>0$. Under Assumptions A1-A8, as $n \rightarrow \infty, \sqrt{n}\left(\gamma_{m}, \hat{\theta}_{n}-\theta_{0}\right)^{\prime} \stackrel{d}{\Rightarrow} \mathcal{N}(0, \Xi)$ where

$$
\Xi=\left(\begin{array}{cc}
\Sigma_{\gamma_{m}} & \Sigma_{\gamma_{m}, \hat{\theta}_{n}} \\
\Sigma_{\gamma_{m}, \hat{\theta}_{n}}^{\prime} & \Sigma_{\hat{\theta}_{n}}
\end{array}\right)
$$

with $\Sigma_{\gamma_{m}}=\left\{\Gamma\left(\ell, \ell^{\prime}\right)\right\}_{1 \leq \ell, \ell^{\prime} \leq m}, \Sigma_{\gamma_{m}, \hat{\theta}_{n}}^{\prime}=\operatorname{Cov}\left(\sqrt{n} J^{-1} Y_{n}, \sqrt{n} \gamma_{m}\right)$ and $\Sigma_{\hat{\theta}_{n}}=$ $\lim _{n \rightarrow \infty} \operatorname{Var}\left(\sqrt{n} J^{-1} Y_{n}\right)=J^{-1} I J^{-1}$ and $Y_{n}$ is given by (15) in the proof of this Theorem. The matrices $I$ and $J$ are defined in Section 3.

Remark 4.1. FRZ considered the univariate case $d=1$. In their paper, they used the LSE and they obtained that

$$
\Sigma_{\gamma_{m}}=\sum_{h=-\infty}^{+\infty}\left\{E\left(e_{t} e_{t-\ell} e_{t-h} e_{t-\ell^{\prime}-h}\right)\right\}_{1 \leq \ell, \ell^{\prime} \leq m}
$$

and denoted it by $\Gamma_{m, m^{\prime}}=\left\{\Gamma\left(\ell, \ell^{\prime}\right)\right\}_{1 \leq \ell, \ell^{\prime} \leq m}$. They introduce the vectors $\lambda_{i}=$ $\left(-\phi_{i-1}^{*}, \ldots,-\phi_{i-p}^{*}, \varphi_{i-1}^{*}, \ldots, \varphi_{i-q}^{*}\right)^{\prime} \in \mathbb{R}^{p+q}$, with the convention $\phi_{i}^{*}=\varphi_{i}^{*}=0$ when $i<0$ and where $\phi_{h}^{*}$ and $\varphi_{h}^{*}$ denote the coefficients defined by

$$
A_{\theta}^{-1}(z)=\sum_{h=0}^{\infty} \phi_{h}^{*} z^{h}, \quad B_{\theta}^{-1}(z)=\sum_{h=0}^{\infty} \varphi_{h}^{*} z^{h}, \quad|z| \leq 1 \quad \text { for } \quad h \geq 0 .
$$

They also introduce the $(p+q) \times m$ matrices $\Lambda_{m}=\left(\lambda_{1}, \ldots, \lambda_{m}\right)$. Using the QMLE, their result gives

$$
\Sigma_{\hat{\theta}_{n}}=\left(\Lambda_{\infty} \Lambda_{\infty}^{\prime}\right)^{-1} \sigma_{e}^{-4} \Lambda_{\infty} \Gamma_{\infty, \infty} \Lambda_{\infty}^{\prime}\left(\Lambda_{\infty} \Lambda_{\infty}^{\prime}\right)^{-1}
$$


where $\sigma_{e}^{2}$ is the variance of the univariate process $e_{t}$. Using the fact that

$$
\frac{\partial e_{t}\left(\theta_{0}\right)}{\partial \theta}=\sum_{i \geq 1} \lambda_{i} e_{t-i}\left(\theta_{0}\right)
$$

we also have

$$
\Sigma_{\gamma_{m}, \hat{\theta}_{n}}^{\prime}=-\sigma_{e}^{-2}\left(\Lambda_{\infty} \Lambda_{\infty}^{\prime}\right)^{-1} \Lambda_{\infty} \Gamma_{\infty, m},
$$

which are the expressions given in Theorem 1 of FRZ.

\section{Asymptotic distribution of residual empirical autocovariances and autocorrelations}

Denoting the diagonal matrices by

$$
S_{e}=\operatorname{Diag}\left(\sigma_{e}(1), \ldots, \sigma_{e}(d)\right) \quad \text { and } \quad \hat{S}_{e}=\operatorname{Diag}\left(\hat{\sigma}_{e}(1), \ldots, \hat{\sigma}_{e}(d)\right),
$$

where $\sigma_{e}^{2}(i)$ is the variance of the $i$-th coordinate of $e_{t}$ and $\hat{\sigma}_{e}^{2}(i)$ is its sample estimate $\left(\right.$ i.e. $\sigma_{e}(i)=\sqrt{E e_{i t}^{2}}$ and $\left.\hat{\sigma}_{e}(i)=\sqrt{n^{-1} \sum_{t=1}^{n} \hat{e}_{i t}^{2}}\right)$. The theoretical and sample autocorrelations at lag $\ell$ are respectively defined by $R_{e}(\ell)=$ $S_{e}^{-1} \Gamma_{e}(\ell) S_{e}^{-1}$ and $\hat{R}_{e}(\ell)=\hat{S}_{e}^{-1} \hat{\Gamma}_{e}(\ell) \hat{S}_{e}^{-1}$, with $\Gamma_{e}(\ell):=E e_{t} e_{t-\ell}^{\prime}=0$ for all $\ell \neq 0$. Consider the vector of the first $m$ sample autocorrelations

$$
\hat{\rho}_{m}=\left(\left\{\operatorname{vec} \hat{R}_{e}(1)\right\}^{\prime}, \ldots,\left\{\operatorname{vec} \hat{R}_{e}(m)\right\}^{\prime}\right)^{\prime} .
$$

The following Theorem gives the limiting distribution of the residual autocovariances and autocorrelations.

Theorem 5.1. Under the none Assumptions as in Theorem 4.1,

$$
\begin{aligned}
& \sqrt{n} \hat{\Gamma}_{m} \Rightarrow \mathcal{N}\left(0, \Sigma_{\hat{\Gamma}_{m}}\right) \quad \text { and } \quad \sqrt{n} \hat{\rho}_{m} \Rightarrow \mathcal{N}\left(0, \Sigma_{\hat{\rho}_{m}}\right) \quad \text { where } \\
& \Sigma_{\hat{\Gamma}_{m}}=\Sigma_{\gamma_{m}}+\Phi_{m} \Sigma_{\hat{\theta}_{n}} \Phi_{m}^{\prime}+\Phi_{m} \Sigma_{\hat{\theta}_{n}, \gamma_{m}}+\Sigma_{\hat{\theta}_{n}, \gamma_{m}}^{\prime} \Phi_{m}^{\prime} \\
& \Sigma_{\hat{\rho}_{m}}=\left\{I_{m} \otimes\left(S_{e} \otimes S_{e}\right)^{-1}\right\} \Sigma_{\hat{\Gamma}_{m}}\left\{I_{m} \otimes\left(S_{e} \otimes S_{e}\right)^{-1}\right\}
\end{aligned}
$$

and $\Phi_{m}$ is given by (18) in the proof of this Theorem.

Chabot-Hallé and Duchesne (2008) obtained a similar result under a difference martingale assumption on the noises. 
Remark 5.1. Considered the univariate case $d=1$, as in FRZ. We obtain

$$
\begin{aligned}
\Phi_{m} & =E\left\{\left(\begin{array}{c}
e_{t-1} \\
\vdots \\
e_{t-m}
\end{array}\right) \otimes \frac{\partial e_{t}^{\prime}\left(\theta_{0}\right)}{\partial \theta}\right\}=\sum_{i \geq 1} E\left\{\left(\begin{array}{c}
e_{t-1} \\
\vdots \\
e_{t-m}
\end{array}\right) e_{t-i} \lambda_{i}^{\prime}\right\} \\
& =\sum_{i=1}^{m} E\left\{\left(\begin{array}{c}
e_{t-1} e_{t-i} \\
\vdots \\
e_{t-m} e_{t-i}
\end{array}\right) \lambda_{i}^{\prime}\right\}=\sigma_{e}^{2} \Lambda_{m}^{\prime} .
\end{aligned}
$$

Using this notation and in view of Remark 4.1, Theorem 5.1 gives

$$
\begin{aligned}
\Sigma_{\hat{\Gamma}_{m}}= & \Gamma_{m, m}+\Lambda_{m}^{\prime}\left(\Lambda_{\infty} \Lambda_{\infty}^{\prime}\right)^{-1} \Lambda_{\infty} \Gamma_{\infty, \infty} \Lambda_{\infty}^{\prime}\left(\Lambda_{\infty} \Lambda_{\infty}^{\prime}\right)^{-1} \Lambda_{m} \\
& -\Lambda_{m}^{\prime}\left(\Lambda_{\infty} \Lambda_{\infty}^{\prime}\right)^{-1} \Lambda_{\infty} \Gamma_{\infty, m}-\Gamma_{m, \infty} \Lambda_{\infty}^{\prime}\left(\Lambda_{\infty} \Lambda_{\infty}^{\prime}\right)^{-1} \Lambda_{m},
\end{aligned}
$$

and $\Sigma_{\hat{\rho}_{m}}=\sigma_{e}^{-4} \Sigma_{\hat{\Gamma}_{m}}$. This last is the result given in Theorem 2 of FRZ.

Remark 5.2. In the standard strong VARMA case, i.e. when $\mathbf{A} 4$ is replaced by the assumption that $\left(\epsilon_{t}\right)$ is an iid sequence, we have $\Sigma_{\gamma_{m}}=I_{m} \otimes \Sigma_{e 0} \otimes \Sigma_{e 0}$, $\Sigma_{\hat{\theta}_{n}}=2 J^{-1}$ (because $I=2 J$ ) and

$$
\begin{aligned}
\Sigma_{\hat{\theta}_{n}, \gamma_{m}}^{\prime} & =-2 E\left\{\left(\begin{array}{c}
e_{t-1} \\
\vdots \\
e_{t-m}
\end{array}\right) \otimes e_{t}\right\}\left\{e_{t}^{\prime} \Sigma_{e 0}^{-1} \frac{\partial e_{t}\left(\theta_{0}\right)}{\partial \theta^{\prime}} J^{-1}\right\} \\
& =-E\left\{\left[\left(\begin{array}{c}
e_{t-1} \\
\vdots \\
e_{t-m}
\end{array}\right) \otimes \frac{\partial e_{t}\left(\theta_{0}\right)}{\partial \theta^{\prime}}\right]\left(2 J^{-1}\right)\right\}=-\Phi_{m} \Sigma_{\hat{\theta}_{n}} .
\end{aligned}
$$

Thus $\Sigma_{\hat{\Gamma}_{m}}=I_{m} \otimes \Sigma_{e 0} \otimes \Sigma_{e 0}-\Phi_{m} \Sigma_{\hat{\theta}_{n}} \Phi_{m}^{\prime}$, that the result obtained by Hosking (1981b), Chabot-Hallé and Duchesne (2008).

\section{Limiting distribution of the portmanteau statistics}

Box and Pierce (1970) (BP hereafter) derived a goodness-of-fit test, the portmanteau test, for univariate strong ARMA models. Ljung and Box (1978) (LB hereafter) proposed a modified portmanteau test which is nowadays one of the most popular diagnostic checking tools in ARMA modeling of time series. The multivariate version of the $\mathbf{B P}$ portmanteau statistic 
was introduced by Chitturi (1974). Hosking (1981a) gave several equivalent forms of this statistic. Basic forms are

$$
\begin{aligned}
Q_{m} & =n \sum_{h=1}^{m} \operatorname{Tr}\left(\hat{\Gamma}_{e}^{\prime}(h) \hat{\Gamma}_{e}^{-1}(0) \hat{\Gamma}_{e}(h) \hat{\Gamma}_{e}^{-1}(0)\right) \\
& =n \sum_{h=1}^{m} \operatorname{vec}\left(\hat{\Gamma}_{e}(h)\right)^{\prime}\left(\hat{\Gamma}_{e}^{-1}(0) \otimes I_{d}\right) \operatorname{vec}\left(\hat{\Gamma}_{e}^{-1}(0) \hat{\Gamma}_{e}(h)\right) \\
& =n \sum_{h=1}^{m} \operatorname{vec}\left(\hat{\Gamma}_{e}(h)\right)^{\prime}\left(\hat{\Gamma}_{e}^{-1}(0) \otimes I_{d}\right)\left(I_{d} \otimes \hat{\Gamma}_{e}^{-1}(0)\right) \operatorname{vec}\left(\hat{\Gamma}_{e}(h)\right) \\
& =n \sum_{h=1}^{m} \operatorname{vec}\left(\hat{\Gamma}_{e}(h)\right)^{\prime}\left(\hat{\Gamma}_{e}^{-1}(0) \otimes \hat{\Gamma}_{e}^{-1}(0)\right) \operatorname{vec}\left(\hat{\Gamma}_{e}(h)\right) \\
& =n \hat{\Gamma}_{m}^{\prime}\left(I_{m} \otimes\left\{\hat{\Gamma}_{e}^{-1}(0) \otimes \hat{\Gamma}_{e}^{-1}(0)\right\}\right) \hat{\Gamma}_{m} \\
& =n \hat{\rho}_{m}^{\prime}\left(I_{m} \otimes\left\{\hat{\Gamma}_{e}(0) \hat{\Gamma}_{e}^{-1}(0) \hat{\Gamma}_{e}(0)\right\} \otimes\left\{\hat{\Gamma}_{e}(0) \hat{\Gamma}_{e}^{-1}(0) \hat{\Gamma}_{e}(0)\right\}\right) \hat{\rho}_{m} \\
& =n \hat{\rho}_{m}^{\prime}\left(I_{m} \otimes\left\{\hat{R}_{e}^{-1}(0) \otimes \hat{R}_{e}^{-1}(0)\right\}\right) \hat{\rho}_{m} .
\end{aligned}
$$

Where the equalities is obtained from the elementary identities $\operatorname{vec}(A B)=$ $(I \otimes A) \operatorname{vec} B,(A \otimes B)(C \otimes D)=A C \otimes B D$ and $\operatorname{Tr}(A B C)=\operatorname{vec}\left(A^{\prime}\right)^{\prime}\left(C^{\prime} \otimes\right.$ $I) \operatorname{vec} B$. As for the univariate LB portmanteau statistic, Hosking (1980) defined the modified portmanteau statistic

$$
\tilde{Q}_{m}=n^{2} \sum_{h=1}^{m}(n-h)^{-1} \operatorname{Tr}\left(\hat{\Gamma}_{e}^{\prime}(h) \hat{\Gamma}_{e}^{-1}(0) \hat{\Gamma}_{e}(h) \hat{\Gamma}_{e}^{-1}(0)\right) .
$$

These portmanteau statistics are generally used to test the null hypothesis

$$
H_{0}:\left(X_{t}\right) \text { satisfies a } \operatorname{VARMA}(p, q) \text { representation }
$$

against the alternative

$H_{1}: \quad\left(X_{t}\right)$ does not admit a VARMA representation or admits a $\operatorname{VARMA}(P, Q)$ representation with $P>p$ or $Q>q$.

These portmanteau tests are very useful tools for checking the overall significance of the residual autocorrelations. Under the assumption that the data generating process (DGP) follows a strong $\operatorname{VARMA}(p, q)$ model, the 
asymptotic distribution of the statistics $Q_{m}$ and $\tilde{Q}_{m}$ is generally approximated by the $\chi_{d^{2} m-k_{0}}^{2}$ distribution $\left(d^{2} m>k_{0}\right)$. When the innovations are gaussian, Hosking (1980) found that the finite-sample distribution of $\tilde{Q}_{m}$ is more nearly to $\chi_{d^{2}(m-(p+q))}^{2}$ than that of $Q_{m}$. From Theorem 5.1 we deduce the following result, in the case of weak $\operatorname{VARMA}(p, q)$ models, which gives the exact asymptotic distribution of the standard portmanteau statistics $Q_{m}$. We will see that the distribution may be very different from the $\chi_{d^{2} m-k_{0}}^{2}$ in the case of strong $\operatorname{VARMA}(p, q)$ models.

Theorem 6.1. Under Assumptions in Theorem 5.1, the statistics $Q_{m}$ and $\tilde{Q}_{m}$ converge in distribution, as $n \rightarrow \infty$, to

$$
Z_{m}\left(\xi_{m}\right)=\sum_{i=1}^{d^{2} m} \xi_{i, d^{2} m} Z_{i}^{2}
$$

where $\xi_{m}=\left(\xi_{1, d^{2} m}, \ldots, \xi_{d^{2} m, d^{2} m}\right)^{\prime}$ is the vector of the eigenvalues of the matrix

$$
\Omega_{m}=\left(I_{m} \otimes \Sigma_{e}^{-1 / 2} \otimes \Sigma_{e}^{-1 / 2}\right) \Sigma_{\hat{\Gamma}_{m}}\left(I_{m} \otimes \Sigma_{e}^{-1 / 2} \otimes \Sigma_{e}^{-1 / 2}\right)
$$

and $Z_{1}, \ldots, Z_{d^{2} m}$ are independent $\mathcal{N}(0,1)$ variables.

Francq and Raïssi (2007) considered the sub-class of a weak vector autoregressive (VAR). They obtained a similar result and showed that the $\chi_{d^{2}(m-p)}^{2}$ approximation is no longer valid in the weak $\operatorname{VAR}(p)$ cases. Considering the univariate ARMA case, $d=1$, we retrieve exactly the result given in Theorem 3 of FRZ.

It is seen in Theorem 6.1, that the asymptotic distribution of the $\mathbf{B P}$ and $\mathbf{L B}$ portmanteau tests depends on the nuisance parameters involving in $\Sigma_{e}$, the matrix $\Phi_{m}$ and the elements of the matrix $\Xi$. We need a consistent estimator of the above unknown matrices. The matrix $\Sigma_{e}$ can be consistently estimated by its sample estimate $\hat{\Sigma}_{e}=\hat{\Gamma}_{e}(0)$. The matrix $\Phi_{m}$ can be easily estimated by its empirical counterpart

$$
\hat{\Phi}_{m}=\frac{1}{n} \sum_{t=1}^{n}\left\{\left(\hat{e}_{t-1}^{\prime}, \ldots, \hat{e}_{t-m}^{\prime}\right)^{\prime} \otimes \frac{\partial e_{t}\left(\theta_{0}\right)}{\partial \theta^{\prime}}\right\}_{\theta_{0}=\hat{\theta}_{n}} .
$$

In the econometric literature the nonparametric kernel estimator, also called heteroskedastic autocorrelation consistent (HAC) estimator (see Newey and West, 1987, or Andrews, 1991), is widely used to estimate covariance matrices 
of the form $\Xi$. Interpreting $(2 \pi)^{-1} \Xi$ as the spectral density of the stationary process $\left(\Upsilon_{t}\right)$ evaluated at frequency 0 (see Brockwell and Davis, 1991, p. 459), an alternative method consists in using a parametric AR estimate of the spectral density of $\Upsilon_{t}=\left(\Upsilon_{1, t}^{\prime}, \Upsilon_{2, t}^{\prime}\right)^{\prime}$, where $\Upsilon_{1, t}=\left(e_{t-1}^{\prime}, \ldots, e_{t-m}^{\prime}\right)^{\prime} \otimes e_{t}$ and $\Upsilon_{2, t}=-2 J^{-1}\left(\partial e_{t}^{\prime}\left(\theta_{0}\right) / \partial \theta\right) \Sigma_{e 0}^{-1} e_{t}\left(\theta_{0}\right)$. This approach, which has been studied by Berk (1974) (see also den Hann and Levin, 1997), rests on the expression

$$
\Xi=\boldsymbol{\Phi}^{-1}(1) \Sigma_{u} \boldsymbol{\Phi}^{\prime-1}(1)
$$

when $\left(\Upsilon_{t}\right)$ satisfies an $\operatorname{AR}(\infty)$ representation of the form

$$
\boldsymbol{\Phi}(L) \Upsilon_{t}:=\Upsilon_{t}+\sum_{i=1}^{\infty} \Phi_{i} \Upsilon_{t-i}=u_{t}
$$

where $u_{t}$ is a weak white noise with variance matrix $\Sigma_{u}$. Since $\Upsilon_{t}$ is not observable, let $\hat{\Upsilon}_{t}$ be the vector obtained by replacing $\theta_{0}$ by $\hat{\theta}_{n}$ in $\Upsilon_{t}$. Let $\hat{\boldsymbol{\Phi}}_{r}(z)=\mathrm{I}_{k_{0}+d^{2} m}+\sum_{i=1}^{r} \hat{\Phi}_{r, i} z^{i}$, where $\hat{\Phi}_{r, 1}, \ldots, \hat{\Phi}_{r, r}$ denote the coefficients of the LS regression of $\hat{\Upsilon}_{t}$ on $\hat{\Upsilon}_{t-1}, \ldots, \hat{\Upsilon}_{t-r}$. Let $\hat{u}_{r, t}$ be the residuals of this regression, and let $\hat{\Sigma}_{\hat{u}_{r}}$ be the empirical variance of $\hat{u}_{r, 1}, \ldots, \hat{u}_{r, n}$.

We are now able to state the following Theorem, which is an extension of a result given in FRZ.

Theorem 6.2. In addition to the assumptions of Theorem 6.1, assume that the process $\left(\Upsilon_{t}\right)$ admits an $A R(\infty)$ representation (7) in which the roots of $\operatorname{det} \Phi(z)=0$ are outside the unit disk, $\left\|\Phi_{i}\right\|=o\left(i^{-2}\right)$, and $\Sigma_{u}=\operatorname{Var}\left(u_{t}\right)$ is non-singular. Moreover we assume that $E\left\|\epsilon_{t}\right\|^{8+4 \nu}<\infty$ and $\sum_{k=0}^{\infty}\left\{\alpha_{X, \epsilon}(k)\right\}^{\nu /(2+\nu)}<\infty$ for some $\nu>0$, where $\left\{\alpha_{X, \epsilon}(k)\right\}_{k \geq 0}$ denotes the sequence of the strong mixing coefficients of the process $\left(X_{t}^{\prime}, \epsilon_{t}^{\prime}\right)^{\prime}$. Then, the spectral estimator of $\Xi$

$$
\hat{\Xi}^{\mathrm{SP}}:=\hat{\boldsymbol{\Phi}}_{r}^{-1}(1) \hat{\Sigma}_{\hat{u}_{r}} \hat{\boldsymbol{\Phi}}_{r}^{\prime-1}(1) \rightarrow \Xi
$$

in probability when $r=r(n) \rightarrow \infty$ and $r^{3} / n \rightarrow 0$ as $n \rightarrow \infty$.

Let $\hat{\Omega}_{m}$ be the matrix obtained by replacing $\Xi$ by $\hat{\Xi}$ and $\Sigma_{e}$ by $\hat{\Sigma}_{e}$ in $\Omega_{m}$. Denote by $\hat{\xi}_{m}=\left(\hat{\xi}_{1, d^{2} m}, \ldots, \hat{\xi}_{d^{2} m, d^{2} m}\right)^{\prime}$ the vector of the eigenvalues of $\hat{\Omega}_{m}$. At the asymptotic level $\alpha$, the $\mathbf{L B}$ test (resp. the $\mathbf{B P}$ test) consists in rejecting the adequacy of the weak $\operatorname{VARMA}(p, q)$ model when

$$
\tilde{Q}_{m}>S_{m}(1-\alpha) \quad\left(\operatorname{resp} . \quad Q_{m}>S_{m}(1-\alpha)\right)
$$

where $S_{m}(1-\alpha)$ is such that $P\left\{Z_{m}\left(\hat{\xi}_{m}\right)>S_{m}(1-\alpha)\right\}=\alpha$. 


\section{Numerical illustrations}

In this section, we present the models we simulate and we give the steps to implement the modified version of the portmanteau test. By means of Monte Carlo experiments, we investigate the finite sample properties of the test introduced in this paper.

\subsection{Simulating models}

To generate the strong and the weak VARMA models, we consider the following bivariate $\operatorname{VARMA}(1,1)$ model in echelon form

$$
\begin{aligned}
\left(\begin{array}{c}
X_{1, t} \\
X_{2, t}
\end{array}\right)= & \left(\begin{array}{cc}
0 & 0 \\
0 & a_{1}(2,2)
\end{array}\right)\left(\begin{array}{l}
X_{1, t-1} \\
X_{2, t-1}
\end{array}\right)+\left(\begin{array}{c}
\epsilon_{1, t} \\
\epsilon_{2, t}
\end{array}\right) \\
& -\left(\begin{array}{cc}
0 & 0 \\
b_{1}(2,1) & b_{1}(2,2)
\end{array}\right)\left(\begin{array}{c}
\epsilon_{1, t-1} \\
\epsilon_{2, t-1}
\end{array}\right),
\end{aligned}
$$

where $\left(a_{1}(2,2), b_{1}(2,1), b_{1}(2,2)\right)=(0.950,-0.313,0.250)$ and $\epsilon_{t}=\left(\epsilon_{1, t}, \epsilon_{2, t}\right)^{\prime}$ follows a strong or weak white noise.

\subsection{Implementation of the goodness-of-fit portmanteau tests}

Let $X_{1}, \ldots, X_{n}$, be observations of a $d$-multivariate process. For testing the adequacy of a weak $\operatorname{VARMA}(p, q)$ model, we implement the modified version of the portmanteau test, using the following steps:

1. Compute the estimates $\hat{A}_{1}, \ldots, \hat{A}_{p}, \hat{B}_{1}, \ldots, \hat{B}_{q}$ by QMLE.

2. Compute the QMLE residuals $\hat{e}_{t}=\tilde{e}_{t}\left(\hat{\theta}_{n}\right)$ when $p>0$ or $q>0$, and let $\hat{e}_{t}=e_{t}=X_{t}$ when $p=q=0$. When $p+q \neq 0$, we have $\hat{e}_{t}=0$ for $t \leq 0$ and $t>n$ and

$$
\hat{e}_{t}=X_{t}-\sum_{i=1}^{p} A_{0}^{-1}\left(\hat{\theta}_{n}\right) A_{i}\left(\hat{\theta}_{n}\right) \hat{X}_{t-i}+\sum_{i=1}^{q} A_{0}^{-1}\left(\hat{\theta}_{n}\right) B_{i}\left(\hat{\theta}_{n}\right) B_{0}^{-1}\left(\hat{\theta}_{n}\right) A_{0}\left(\hat{\theta}_{n}\right) \hat{e}_{t-i},
$$

for $t=1, \ldots, n$, with $\hat{X}_{t}=0$ for $t \leq 0$ and $\hat{X}_{t}=X_{t}$ for $t \geq 1$.

3. Compute the residual autocovariances $\hat{\Gamma}_{e}(0)=\hat{\Sigma}_{e}$ and $\hat{\Gamma}_{e}(h)$ for $h=$ $1, \ldots, m$ and $\hat{\Gamma}_{m}=\left(\left\{\hat{\Gamma}_{e}(1)\right\}^{\prime}, \ldots,\left\{\hat{\Gamma}_{e}(m)\right\}^{\prime}\right)^{\prime}$.

4. Compute the matrix $\hat{J}=2 n^{-1} \sum_{t=1}^{n}\left(\partial \hat{e}_{t}^{\prime} / \partial \theta\right) \hat{\Sigma}_{e 0}^{-1}\left(\partial \hat{e}_{t} / \partial \theta^{\prime}\right)$. 
5. Compute $\hat{\Upsilon}_{t}=\left(\hat{\Upsilon}_{1, t}^{\prime}, \hat{\Upsilon}_{2, t}^{\prime}\right)^{\prime}$, where $\hat{\Upsilon}_{1, t}=\left(\hat{e}_{t-1}^{\prime}, \ldots, \hat{e}_{t-m}^{\prime}\right)^{\prime} \otimes \hat{e}_{t}$ and $\hat{\Upsilon}_{2, t}=-2 \hat{J}^{-1}\left(\partial \hat{e}_{t}^{\prime} / \partial \theta\right) \hat{\Sigma}_{e}^{-1} \hat{e}_{t}$.

6. Fit the $\operatorname{VAR}(r)$ model

$$
\hat{\boldsymbol{\Phi}}_{r}(L) \hat{\Upsilon}_{t}:=\left(I_{d^{2} m+k_{0}}+\sum_{i=1}^{r} \hat{\Phi}_{r, i}(L)\right) \hat{\Upsilon}_{t}=\hat{u}_{r, t}
$$

The VAR order $r$ can be fixed in a strong VARMA case or selected by $\mathrm{AIC} / \mathrm{BIC}$ information criteria in a weak VARMA case.

7. Use the spectral estimator

$\hat{\Xi}^{\mathrm{SP}}:=\hat{\boldsymbol{\Phi}}_{r}^{-1}(1) \hat{\Sigma}_{\hat{u}_{r}} \hat{\boldsymbol{\Phi}}_{r}^{\prime-1}(1)=\left(\begin{array}{cc}\hat{\Sigma}_{\gamma_{m}} & \hat{\Sigma}_{\gamma_{m}, \hat{\theta}_{n}} \\ \hat{\Sigma}_{\gamma_{m}, \hat{\theta}_{n}}^{\prime} & \hat{\Sigma}_{\hat{\theta}_{n}}\end{array}\right), \hat{\Sigma}_{\hat{u}_{r}}=\frac{1}{n} \sum_{t=1}^{n} \hat{u}_{r, t} \hat{u}_{r, t}^{\prime}$,

defined in Theorem 6.2.

8. Define the estimator

$$
\hat{\Phi}_{m}=\frac{1}{n} \sum_{t=1}^{n}\left\{\left(\hat{e}_{t-1}^{\prime}, \ldots, \hat{e}_{t-m}^{\prime}\right)^{\prime} \otimes \frac{\partial e_{t}\left(\theta_{0}\right)}{\partial \theta^{\prime}}\right\}_{\theta_{0}=\hat{\theta}_{n}} .
$$

9. Define the estimators

$$
\begin{aligned}
& \hat{\Sigma}_{\hat{\Gamma}_{m}}=\hat{\Sigma}_{\gamma_{m}}+\hat{\Phi}_{m} \hat{\Sigma}_{\hat{\theta}_{n}} \hat{\Phi}_{m}^{\prime}+\hat{\Phi}_{m} \hat{\Sigma}_{\hat{\theta}_{n}, \gamma_{m}}+\hat{\Sigma}_{\hat{\theta}_{n}, \gamma_{m}}^{\prime} \hat{\Phi}_{m}^{\prime} \\
& \hat{\Sigma}_{\hat{\rho}_{m}}=\left\{I_{m} \otimes\left(\hat{S}_{e} \otimes \hat{S}_{e}\right)^{-1}\right\} \hat{\Sigma}_{\hat{\Gamma}_{m}}\left\{I_{m} \otimes\left(\hat{S}_{e} \otimes \hat{S}_{e}\right)^{-1}\right\} .
\end{aligned}
$$

10. Compute the eigenvalues $\hat{\xi}_{m}=\left(\hat{\xi}_{1, d^{2} m}, \ldots, \hat{\xi}_{d^{2} m, d^{2} m}\right)^{\prime}$ of the matrix

$$
\hat{\Omega}_{m}=\left(I_{m} \otimes \hat{\Sigma}_{e}^{-1 / 2} \otimes \hat{\Sigma}_{e}^{-1 / 2}\right) \hat{\Sigma}_{\hat{\Gamma}_{m}}\left(I_{m} \otimes \hat{\Sigma}_{e}^{-1 / 2} \otimes \hat{\Sigma}_{e}^{-1 / 2}\right) .
$$

11. Compute the portmanteau statistics

$$
\begin{aligned}
& Q_{m}=n \hat{\rho}_{m}^{\prime}\left(I_{m} \otimes\left\{\hat{R}_{e}^{-1}(0) \otimes \hat{R}_{e}^{-1}(0)\right\}\right) \hat{\rho}_{m} \text { and } \\
& \tilde{Q}_{m}=n^{2} \sum_{h=1}^{m} \frac{1}{(n-h)} \operatorname{Tr}\left(\hat{\Gamma}_{e}^{\prime}(h) \hat{\Gamma}_{e}^{-1}(0) \hat{\Gamma}_{e}(h) \hat{\Gamma}_{e}^{-1}(0)\right)
\end{aligned}
$$


12. Evaluate the $p$-values

$$
P\left\{Z_{m}\left(\hat{\xi}_{m}\right)>Q_{m}\right\} \text { and } P\left\{Z_{m}\left(\hat{\xi}_{m}\right)>\tilde{Q}_{m}\right\}, Z_{m}\left(\hat{\xi}_{m}\right)=\sum_{i=1}^{d^{2} m} \hat{\xi}_{i, d^{2} m} Z_{i}^{2},
$$

using the Imhof algorithm (1961). The BP test (resp. the LB test) rejects the adequacy of the weak $\operatorname{VARMA}(p, q)$ model when the first (resp. the second) $p$-value is less than the asymptotic level $\alpha$.

\subsection{Empirical size}

In this paper, we only present the results of the modified and standard versions of the $\mathbf{L B}$ test. The results concerning the BP test are not presented here, because they are very close to those of the LB test. The numerical illustrations of this section are made with the softwares $\mathrm{R}$ (see http://cran.r-project.org/) and FORTRAN (to compute the $p$-values using the Imohf algorithm, 1961).

For the nominal level $\alpha=5 \%$, the empirical size over the $N=1,000$ independent replications should vary between the significant limits $3.6 \%$ and $6.4 \%$ with probability $95 \%$. When the relative rejection frequencies are outside the significant limits, they are displayed in bold type in Tables 1, 2, 3 and 4 .

\subsubsection{Strong VARMA model case}

We first consider the strong VARMA case. To generate this model, we assume that in $(8)$ the innovation process $\left(\epsilon_{t}\right)$ is defined by

$$
\left(\begin{array}{c}
\epsilon_{1, t} \\
\epsilon_{2, t}
\end{array}\right) \sim \operatorname{IID} \mathcal{N}\left(0, I_{2}\right)
$$

We simulated $N=1,000$ independent trajectories of size $n=100, n=500$ and $n=2,000$ of Model (8) with the strong gaussian noise (9). For each of these $N$ replications we estimated the coefficients $\left(a_{1}(2,2), b_{1}(2,1), b_{1}(2,2)\right)$, using the Gaussian maximum likelihood estimation method, and we applied portmanteau tests to the residuals for different values of $m$, where $m$ is the number of autocorrelations used in the portmanteau test statistic. For the standard LB test, the model is therefore rejected when the statistic $\tilde{Q}_{m}$ is greater than $\chi_{(4 m-3)}^{2}(0.95)$. This corresponds to a nominal asymptotic

level $\alpha=5 \%$ in the standard case. We know that the asymptotic level of 
the standard LB test is indeed $\alpha=5 \%$ when $\left(a_{1}(2,2), b_{1}(2,1), b_{1}(2,2)\right)=$ $(0,0,0)$. Note however that, even when the noise is strong, the asymptotic level is not exactly $\alpha=5 \%$ when $\left.\left(a_{1}(2,2), b_{1}(2,1), b_{1}(2,2)\right) \neq(0,0,0)\right)$.

For the modified LB test, the model is rejected when the statistic $\tilde{Q}_{m}$ is greater than $S_{m}(0.95)$ i.e. when the $p$-value $\left(P\left\{Z_{m}\left(\hat{\xi}_{m}\right)>\tilde{Q}_{m}\right\}\right)$ is less than the asymptotic level $\alpha=0.05$. Let $A$ and $B$ be the $(2 \times 2)$-matrices with non zero elements $a_{1}(2,2), b_{1}(2,1)$ and $b_{1}(2,2)$. When the roots of $\operatorname{det}\left(I_{2}-\right.$ $A z) \operatorname{det}\left(I_{2}-B z\right)=0$ are near the unit disk, the asymptotic distribution of $\tilde{Q}_{m}$ is likely to be far from its $\chi_{(4 m-3)}^{2}$ approximation. Table 1 displays the relative rejection frequencies of the null hypothesis $H_{0}$ that the DGP follows a $\operatorname{VARMA}(1,1)$, over the $N=1,000$ independent replications. As expected the observed relative rejection frequency of the standard LB test is very far from the nominal $\alpha=5 \%$ when the number $m$ of autocorrelations used in the $\mathbf{L B}$ statistic is small. This is in accordance with the results in the literature on the standard VARMA models. In particular, Hosking (1980) showed that the statistic $\tilde{Q}_{m}$ has approximately the chi-squared distribution $\chi_{d^{2}(m-(p+q))}^{2}$ without any identifiability contraint. The theory that the $\chi_{(4 m-3)}^{2}$ approximation is better for larger $m$ is confirmed. We draw the conclusion that, even in the strong VARMA case, the modified version is preferable to the standard one, when the number $m$ of autocorrelations used is small.

Table 1: Empirical size (in \%) of the standard and modified versions of the LB test in the case of the strong $\operatorname{VARMA}(1,1)$ model $(8)-(9)$.

\begin{tabular}{lrcrccccccr}
\hline \hline & \multicolumn{3}{c}{$m=1$} & \multicolumn{3}{c}{$m=2$} & \multicolumn{3}{c}{$m=3$} \\
Length $n$ & 100 & 500 & 2,000 & 100 & 500 & 2,000 & 100 & 500 & 2,000 \\
modified LB & 5.5 & 5.6 & 4.0 & 3.7 & 4.4 & 4.8 & $\mathbf{2 . 6}$ & 4.1 & $\mathbf{3 . 3}$ \\
standard LB & $\mathbf{1 6 . 2}$ & $\mathbf{1 6 . 3}$ & $\mathbf{1 5 . 5}$ & $\mathbf{8 . 2}$ & $\mathbf{8 . 0}$ & $\mathbf{7 . 7}$ & $\mathbf{6 . 7}$ & $\mathbf{6 . 8}$ & 5.8 \\
\hline & \multicolumn{4}{c}{$m=4$} & \multicolumn{4}{c}{$m=6$} & \multicolumn{4}{c}{$m=10$} \\
Length $n$ & 100 & 500 & 2,000 & 100 & 500 & 2,000 & 100 & 500 & 2,000 \\
modified LB & $\mathbf{2 . 2}$ & 3.9 & 4.4 & $\mathbf{2 . 3}$ & 3.7 & $\mathbf{3 . 4}$ & $\mathbf{6 . 8}$ & 4.2 & $\mathbf{3 . 5}$ \\
standard LB & 5.6 & 6.0 & 6.2 & 5.1 & 5.9 & 4.5 & 5.0 & 5.7 & 4.9 \\
\hline \hline
\end{tabular}

\subsubsection{Weak VARMA model case}

The $\operatorname{GARCH}(p, q)$ models constitute important examples of weak white noises in the univariate case. These models have numerous extensions to the 
multivariate framework (see Bauwens, Laurent and Rombouts (2006) for a review). Jeantheau (1998) has proposed a simple extension of the multivariate $\operatorname{GARCH}(p, q)$ with conditional constant correlation. In this model, the process $\left(\epsilon_{t}\right)$ verifies the following relation $\epsilon_{t}=H_{t} \eta_{t}$ where $\left\{\eta_{t}=\left(\eta_{1, t}, \ldots, \eta_{d, t}\right)^{\prime}\right\}_{t}$ is an iid centered process with $\operatorname{Var}\left\{\eta_{i, t}\right\}=1$ and $H_{t}$ is a diagonal matrix whose elements $h_{i i, t}$ verify

$$
\left(\begin{array}{c}
h_{11, t}^{2} \\
\vdots \\
h_{d d, t}^{2}
\end{array}\right)=\left(\begin{array}{c}
c_{1} \\
\vdots \\
c_{d}
\end{array}\right)+\sum_{i=1}^{q} A_{i}\left(\begin{array}{c}
\epsilon_{1, t-i}^{2} \\
\vdots \\
\epsilon_{d, t-i}^{2}
\end{array}\right)+\sum_{j=1}^{p} B_{j}\left(\begin{array}{c}
h_{11, t-j}^{2} \\
\vdots \\
h_{d d, t-j}^{2}
\end{array}\right)
$$

The elements of the matrices $A_{i}$ and $B_{j}$, as well as the vector $c_{i}$, are supposed to be positive. In addition, suppose that the stationarity conditions hold. For simplicity, we consider the following bivariate $\mathrm{ARCH}(1)$ (i.e. a bivariate $\operatorname{GARCH}(p, q)$ model with $p=0, q=1)$ model

$$
\left(\begin{array}{l}
\epsilon_{1, t} \\
\epsilon_{2, t}
\end{array}\right)=\left(\begin{array}{cc}
h_{11, t} & 0 \\
0 & h_{22, t}
\end{array}\right)\left(\begin{array}{l}
\eta_{1, t} \\
\eta_{2, t}
\end{array}\right)
$$

where

$$
\left(\begin{array}{c}
h_{11, t}^{2} \\
h_{22, t}^{2}
\end{array}\right)=\left(\begin{array}{c}
c_{1} \\
c_{2}
\end{array}\right)+\left(\begin{array}{cc}
a_{11} & 0 \\
a_{21} & a_{22}
\end{array}\right)\left(\begin{array}{c}
\epsilon_{1, t-1}^{2} \\
\epsilon_{2, t-1}^{2}
\end{array}\right) .
$$

We now repeat the same experiment on different weak $\operatorname{VARMA}(1,1)$ models. For the estimation of the coefficients, we used the quasi-maximum likelihood estimation method and we applied portmanteau tests to the residuals for different values of $m$. We first assume that in (8) the innovation process $\left(\epsilon_{t}\right)$ is an $\operatorname{ARCH}(1)$ model defined in equation (10) with $c_{1}=0.3, c_{2}=0.2$, $a_{11}=0.45, a_{21}=0.4$ and $a_{22}=0.25$. In two other sets of experiments, we assume that in (8) the innovation process $\left(\epsilon_{t}\right)$ is defined by

$$
\left(\begin{array}{c}
\epsilon_{1, t} \\
\epsilon_{2, t}
\end{array}\right)=\left(\begin{array}{c}
\eta_{1, t} \eta_{2, t-1} \eta_{1, t-2} \\
\eta_{2, t} \eta_{1, t-1} \eta_{2, t-2}
\end{array}\right), \quad \text { with }\left(\begin{array}{c}
\eta_{1, t} \\
\eta_{2, t}
\end{array}\right) \sim \operatorname{IID} \mathcal{N}\left(0, I_{2}\right),
$$

and then by

$$
\left(\begin{array}{c}
\epsilon_{1, t} \\
\epsilon_{2, t}
\end{array}\right)=\left(\begin{array}{c}
\eta_{1, t}\left(\left|\eta_{1, t-1}\right|+1\right)^{-1} \\
\eta_{2, t}\left(\left|\eta_{2, t-1}\right|+1\right)^{-1}
\end{array}\right), \quad \text { with }\left(\begin{array}{c}
\eta_{1, t} \\
\eta_{2, t}
\end{array}\right) \sim \operatorname{IID} \mathcal{N}\left(0, I_{2}\right)
$$


These noises are direct extensions of the weak noises defined by Romano and Thombs (1996) in the univariate case.

As expected, Tables 2 and 3 show that the standard LB test poorly performs to assess the adequacy of these weak $\operatorname{VARMA}(1,1)$ models. In view of the observed relative rejection frequency, the standard $\mathbf{L B}$ test rejects very often the true $\operatorname{VARMA}(1,1)$ and all the relative rejection frequencies are definitely outside the significant limits. By contrast, the error of first kind is well controlled by the modified version of the LB test. We draw the conclusion that, for these particular weak VARMA models, the modified version is clearly preferable to the standard one. In contrast, Table 4 shows that the error of first kind is well controlled by all the tests in this particular weak VARMA model, except for the standard LB test when $m=1$. The modified version is also slightly preferable to the standard one.

Table 2: Empirical size (in \%) of the standard and modified versions of the LB test in the case of the weak $\operatorname{VARMA}(1,1)$ model $(8)-(10)$.

\begin{tabular}{|c|c|c|c|c|c|c|c|c|c|}
\hline \multirow{4}{*}{$\begin{array}{l}\text { Length } n \\
\text { modified LB } \\
\text { standard LB }\end{array}$} & \multicolumn{3}{|c|}{$m=1$} & \multicolumn{3}{|c|}{$m=2$} & \multicolumn{3}{|c|}{$m=3$} \\
\hline & 500 & 2,000 & 10,000 & 500 & 2,000 & 10,000 & 500 & 2,000 & 10,000 \\
\hline & 6.9 & 8.5 & 7.4 & 5.9 & 6.4 & 6.3 & 4.2 & 6.1 & 5.3 \\
\hline & 38.5 & 39.7 & 43.1 & 32.0 & 38.2 & 42.9 & 27.6 & 35.6 & 42.1 \\
\hline \multirow{4}{*}{$\begin{array}{l}\text { Length } n \\
\text { modified LB } \\
\text { standard LB }\end{array}$} & \multicolumn{3}{|c|}{$m=4$} & \multicolumn{3}{|c|}{$m=6$} & \multicolumn{3}{|c|}{$m=10$} \\
\hline & 500 & 2,000 & 10,000 & 500 & 2,000 & 10,000 & 500 & 2,000 & 10,000 \\
\hline & 3.9 & 4.8 & 5.5 & 3.3 & 3.8 & 6.0 & 2.7 & 3.5 & 3.8 \\
\hline & 24.9 & 32.3 & 39.2 & 21.2 & 27.3 & 32.1 & 17.0 & 21.2 & 25.4 \\
\hline
\end{tabular}

\subsection{Empirical power}

In this part, we simulated $N=1,000$ independent trajectories of size $n=500, n=1,000$ and $n=5,000$ of a weak $\operatorname{VARMA}(2,2)$ defined by

$$
\begin{aligned}
\left(\begin{array}{l}
X_{1, t} \\
X_{2, t}
\end{array}\right)= & \left(\begin{array}{cc}
0 & 0 \\
0 & 0.225
\end{array}\right)\left(\begin{array}{c}
X_{1, t-1} \\
X_{2, t-1}
\end{array}\right)+\left(\begin{array}{cc}
0 & 0 \\
0 & 0.100
\end{array}\right)\left(\begin{array}{c}
X_{1, t-2} \\
X_{2, t-2}
\end{array}\right) \\
& +\left(\begin{array}{c}
\epsilon_{1, t} \\
\epsilon_{2, t}
\end{array}\right)-\left(\begin{array}{cc}
0 & 0 \\
-0.313 & 0.250
\end{array}\right)\left(\begin{array}{c}
\epsilon_{1, t-1} \\
\epsilon_{2, t-1}
\end{array}\right) \\
& -\left(\begin{array}{cc}
0 & 0 \\
-0.140 & -0.160
\end{array}\right)\left(\begin{array}{c}
\epsilon_{1, t-2} \\
\epsilon_{2, t-2}
\end{array}\right)
\end{aligned}
$$


Table 3: Empirical size (in \%) of the standard and modified versions of the LB test in the case of the weak VARMA $(1,1)$ model (8)-(11).

\begin{tabular}{|c|c|c|c|c|c|c|c|c|c|}
\hline \multirow{4}{*}{$\begin{array}{l}\text { Length } n \\
\text { modified LB } \\
\text { standard LB }\end{array}$} & \multicolumn{3}{|c|}{$m=1$} & \multicolumn{3}{|c|}{$m=2$} & \multicolumn{3}{|c|}{$m=3$} \\
\hline & 500 & 2,000 & 10,000 & 500 & 2,000 & 10,000 & 500 & 2,000 & 10,000 \\
\hline & 4.7 & 3.9 & 5.3 & 3.4 & 2.8 & 4.7 & 3.1 & 2.5 & 4.7 \\
\hline & 58.7 & 58.3 & 62.9 & 59.2 & 57.7 & 64.2 & 48.0 & 53.2 & 57.7 \\
\hline \multirow{4}{*}{$\begin{array}{l}\text { Length } n \\
\text { modified LB } \\
\text { standard LB }\end{array}$} & \multicolumn{3}{|c|}{$m=4$} & \multicolumn{3}{|c|}{$m=6$} & \multicolumn{3}{|c|}{$m=10$} \\
\hline & 500 & 2,000 & 10,000 & 500 & 2,000 & 10,000 & 500 & 2,000 & 10,000 \\
\hline & 2.2 & 2.2 & 5.3 & 1.9 & 2.0 & 4.6 & 3.6 & 3.1 & 5.3 \\
\hline & 41.4 & 46.4 & 51.8 & 33.9 & 40.3 & 44.9 & 25.8 & 32.4 & 37.3 \\
\hline
\end{tabular}

where the innovation process $\left(\epsilon_{t}\right)$ is given by $(11)$.

For each of these $N=1,000$ replications we fitted a $\operatorname{VARMA}(1,1)$ model and perform standard and modified LB test based on $m=1, \ldots, 4,6$ and 10 residual autocorrelations. The adequacy of the $\operatorname{VARMA}(1,1)$ model is rejected when the $p$-value is less than $5 \%$. For this particular weak VARMA model, we have seen that the actual level of the standard version is generally much greater than the 5\% nominal level (see Table 3). As in Hong (1996), we will use the empirical critical values obtained under a weak $\operatorname{VARMA}(1,1)$ model (8)-(11) based on $N=1,000$ replications to compare the powers of the two tests on an equal basis. Table 5 displays the relative rejection frequencies of over the $N=1,000$ independent replications. In this example, the standard and modified versions of the LB test have very similar powers. Note that, the empirical critical values strongly depend on the type of weak VARMA which is generated under the null hypothesis. Therefore, this method consisting in adjusting the critical values only works for very specific hypotheses.

\section{Conclusion}

In this paper we derive the asymptotic distribution of residual empirical autocovariances and autocorrelations under weak assumptions on the noise. We establish the asymptotic distribution of the $\mathbf{L B}$ (or $\mathbf{B P}$ ) portmanteau test statistics for structural VARMA models with nonindependent innovations. This asymptotic distribution is quite different from the usual 
Table 4: Empirical size (in \%) of the standard and modified versions of the LB test in the case of the weak VARMA(1,1) model (8)-(12).

\begin{tabular}{|c|c|c|c|c|c|c|c|c|c|}
\hline \multirow{4}{*}{$\begin{array}{l}\text { Length } n \\
\text { modified LB } \\
\text { standard LB }\end{array}$} & \multicolumn{3}{|c|}{$m=1$} & \multicolumn{3}{|c|}{$m=2$} & \multicolumn{3}{|c|}{$m=3$} \\
\hline & 500 & 2,000 & 10,000 & 500 & 2,000 & 10,000 & 500 & 2,000 & 10,000 \\
\hline & 5.0 & 5.1 & 4.3 & 5.2 & 5.0 & 5.0 & 4.3 & 5.6 & 5.4 \\
\hline & 7.7 & 8.1 & 6.4 & 6.6 & 5.6 & 6.2 & 5.3 & 6.1 & 5.6 \\
\hline & \multicolumn{3}{|c|}{$m=4$} & \multicolumn{3}{|c|}{$m=6$} & \multicolumn{3}{|c|}{$m=10$} \\
\hline \multirow{3}{*}{$\begin{array}{l}\text { Length } n \\
\text { modified LB } \\
\text { standard LB }\end{array}$} & 500 & 2,000 & 10,000 & 500 & 2,000 & 10,000 & 500 & 2,000 & 10,000 \\
\hline & 4.2 & 5.6 & 5.2 & 3.9 & 4.4 & 4.8 & 3.8 & 4.1 & 4.9 \\
\hline & 4.8 & 6.3 & 5.5 & 4.6 & 4.7 & 4.9 & 4.8 & 4.3 & 4.9 \\
\hline
\end{tabular}

chi-squared approximation (i.e. $\chi_{d^{2}(m-p-q)}^{2}$ ) used under iid assumptions on the noise. Therefore the modified versions of $\mathbf{L B}$ and BP are more difficult to implement because their critical values have to be computed from the data, whereas those of the standard versions are simply given in a $\chi^{2}$-table.

In Monte Carlo experiments, we demonstrated that the proposed modified portmanteau test statistics have reasonable finite sample performance, at least for the models considered in our study. Under nonindependent errors, it appears that the standard test statistics are generally unreliable, overrejecting severally, while the proposed test statistics offers satisfactory levels in most cases. Even for independent errors, the modified version may be preferable to the standard one, when the number $m$ of autocorrelations is small. Concerning the relative powers of the two versions, we also show that the modified versions of the $\mathbf{L B}$ and $\mathbf{B P}$ tests have similar powers when the critical values are adjusted. Moreover, the error of first kind is well controlled by the modified versions of the $\mathbf{L B}$ and $\mathbf{B P}$ tests. We draw the conclusion that the modified versions are preferable to the standard ones for diagnosing multivariate models under nonindependent errors. 
Table 5: Empirical power (in \%) of the standard and modified versions of the LB test in the case of the weak $\operatorname{VARMA}(2,2)$ model $(13)-(11)$, with critical values adjusted to obtain exactly $5 \%$ empirical sizes under the null hypothesis of the weak $\operatorname{VARMA}(1,1)$ model (8)-(11).

\begin{tabular}{lrcrrcrrrrr}
\hline \hline & \multicolumn{3}{c}{$m=1$} & \multicolumn{3}{c}{$m=2$} & \multicolumn{3}{c}{$m=3$} \\
Length $n$ & 500 & 1,000 & 5,000 & 500 & 1,000 & 5,000 & 500 & 1,000 & 5,000 \\
\cline { 2 - 11 } modified LB & 10.9 & 37.0 & 96.5 & 70.4 & 96.8 & 99.9 & 80.4 & 92.6 & 99.9 \\
standard LB & 9.1 & 14.3 & 99.8 & 54.4 & 87.8 & 100.0 & 53.5 & 87.0 & 100.0 \\
\hline & \multicolumn{3}{c}{$m=4$} & \multicolumn{3}{c}{$m=6$} & & & $m=10$ \\
Length $n$ & 500 & 1,000 & 5,000 & 500 & 1,000 & 5,000 & 500 & 1,000 & 5,000 \\
\cline { 2 - 10 } modified LB & 81.0 & 90.3 & 99.9 & 86.3 & 91.8 & 99.9 & 46.3 & 90.4 & 100.0 \\
standard LB & 50.5 & 86.4 & 100.0 & 50.7 & 87.2 & 100.0 & 45.6 & 87.5 & 100.0 \\
\hline \hline
\end{tabular}

\section{Appendix}

Proof of Theorem 4.1. Let $\tilde{\ell}_{n}\left(\theta, \Sigma_{e}\right)=-2 n^{-1} \log \tilde{\mathrm{L}}_{n}\left(\theta, \Sigma_{e}\right)$. In BMF, it is shown that $\ell_{n}\left(\theta, \Sigma_{e}\right)=\tilde{\ell}_{n}\left(\theta, \Sigma_{e}\right)+o(1)$ a.s, where

$\ell_{n}\left(\theta, \Sigma_{e}\right):=-\frac{2}{n} \log \mathrm{L}_{n}\left(\theta, \Sigma_{e}\right)=\frac{1}{n} \sum_{t=1}^{n}\left\{d \log (2 \pi)+\log \operatorname{det} \Sigma_{e}+e_{t}^{\prime}(\theta) \Sigma_{e}^{-1} e_{t}(\theta)\right\}$,

and where $\left(e_{t}(\theta)\right)$ is given by (4). It is also shown uniformly in $\theta \in \Theta$ that

$$
\frac{\partial \ell_{n}\left(\theta, \Sigma_{e}\right)}{\partial \theta}=\frac{\partial \tilde{\ell}_{n}\left(\theta, \Sigma_{e}\right)}{\partial \theta}+o(1) \quad \text { a.s. }
$$

The same equality holds for the second-order derivatives of $\tilde{\ell}_{n}\left(\theta, \Sigma_{e}\right)$. In view of Theorem 1 in BMF and $\mathbf{A} 5$, we have almost surely $\hat{\theta}_{n} \rightarrow \theta_{0} \in \stackrel{\circ}{\Theta}$. Thus $\partial \tilde{\ell}_{n}\left(\hat{\theta}_{n}, \hat{\Sigma}_{e}\right) / \partial \theta=0$ for sufficiently large $n$, and a standard Taylor expansion of the derivative of $\tilde{\ell}_{n}$ about $\left(\theta_{0}, \Sigma_{e 0}\right)$, taken at $\left(\hat{\theta}_{n}, \hat{\Sigma}_{e}\right)$, yields

$$
\begin{aligned}
0 & =\sqrt{n} \frac{\partial \tilde{\ell}_{n}\left(\hat{\theta}_{n}, \hat{\Sigma}_{e}\right)}{\partial \theta}=\sqrt{n} \frac{\partial \tilde{\ell}_{n}\left(\theta_{0}, \Sigma_{e 0}\right)}{\partial \theta}+\frac{\partial^{2} \tilde{\ell}_{n}\left(\theta^{*}, \Sigma_{e}^{*}\right)}{\partial \theta \partial \theta^{\prime}} \sqrt{n}\left(\hat{\theta}_{n}-\theta_{0}\right) \\
& =\sqrt{n} \frac{\partial \ell_{n}\left(\theta_{0}, \Sigma_{e 0}\right)}{\partial \theta}+\frac{\partial^{2} \ell_{n}\left(\theta_{0}, \Sigma_{e 0}\right)}{\partial \theta \partial \theta^{\prime}} \sqrt{n}\left(\hat{\theta}_{n}-\theta_{0}\right)+o_{P}(1)
\end{aligned}
$$


using arguments given in FZ (proof of Theorem 2), where $\theta^{*}$ is between $\theta_{0}$ and $\hat{\theta}_{n}$, and $\Sigma_{e}^{*}$ is between $\Sigma_{e 0}$ and $\hat{\Sigma}_{e}$, with $\hat{\Sigma}_{e}=n^{-1} \sum_{t=1}^{n} \tilde{e}_{t}\left(\hat{\theta}_{n}\right) \tilde{e}_{t}^{\prime}\left(\hat{\theta}_{n}\right)$. Thus, by standard arguments, we have from (14):

$$
\begin{aligned}
\sqrt{n}\left(\hat{\theta}_{n}-\theta_{0}\right) & =-J^{-1} \sqrt{n} \frac{\partial \ell_{n}\left(\theta_{0}, \Sigma_{e 0}\right)}{\partial \theta}+o_{P}(1) \\
& =J^{-1} \sqrt{n} Y_{n}+o_{P}(1)
\end{aligned}
$$

where

$$
Y_{n}=-\frac{\partial \ell_{n}\left(\theta_{0}, \Sigma_{e 0}\right)}{\partial \theta}=-\frac{1}{n} \sum_{t=1}^{n} \frac{\partial}{\partial \theta}\left\{d \log (2 \pi)+\log \operatorname{det} \Sigma_{e 0}+e_{t}^{\prime}\left(\theta_{0}\right) \Sigma_{e 0}^{-1} e_{t}\left(\theta_{0}\right)\right\}
$$

Showing that the initial values are asymptotically negligible, and using wellknown results on matrix derivatives (see (5) of Appendix A.13 in Lütkepohl, 2005), we have

$$
Y_{n}=-\frac{2}{n} \sum_{t=1}^{n} \frac{\partial e_{t}^{\prime}\left(\theta_{0}\right)}{\partial \theta} \Sigma_{e 0}^{-1} e_{t}\left(\theta_{0}\right) .
$$

Using the elementary relation $\operatorname{vec}(A B C)=\left(C^{\prime} \otimes A\right) \operatorname{vec}(B)$ (see (4) of Appendix A.12 in Lütkepohl, 2005), we have vec $\gamma(\ell)=n^{-1} \sum_{t=\ell+1}^{n} e_{t-\ell} \otimes e_{t}$. It is easily shown that for $\ell, \ell^{\prime} \geq 1$,

$$
\begin{aligned}
\operatorname{Cov}\left(\sqrt{n} \operatorname{vec} \gamma(\ell), \sqrt{n} \operatorname{vec} \gamma\left(\ell^{\prime}\right)\right) & =\frac{1}{n} \sum_{t=\ell+1}^{n} \sum_{t^{\prime}=\ell^{\prime}+1}^{n} E\left(\left\{e_{t-\ell} \otimes e_{t}\right\}\left\{e_{t^{\prime}-\ell^{\prime}} \otimes e_{t^{\prime}}\right\}^{\prime}\right) \\
& \rightarrow \Gamma\left(\ell, \ell^{\prime}\right) \text { as } n \rightarrow \infty
\end{aligned}
$$

Then, we have

$$
\Sigma_{\gamma_{m}}=\left\{\Gamma\left(\ell, \ell^{\prime}\right)\right\}_{1 \leq \ell, \ell^{\prime} \leq m}
$$

By stationarity of $\left(e_{t}\right)$ and $\left(Y_{t}\right)$, we have

$$
\begin{aligned}
\operatorname{Cov}\left(\sqrt{n} J^{-1} Y_{n}, \sqrt{n} \operatorname{vec} \gamma(\ell)\right) & =-\frac{2}{n} \sum_{t=1}^{n} \sum_{t=\ell+1}^{n} J^{-1} \operatorname{Cov}\left(\frac{\partial e_{t}^{\prime}\left(\theta_{0}\right)}{\partial \theta} \Sigma_{e 0}^{-1} e_{t}, e_{t-\ell} \otimes e_{t}\right) \\
& =-\frac{2}{n} \sum_{h=-n+1}^{n-1}(n-|h|) J^{-1} \operatorname{Cov}\left(\frac{\partial e_{t}^{\prime}\left(\theta_{0}\right)}{\partial \theta} \Sigma_{e 0}^{-1} e_{t}, e_{t-h-\ell} \otimes e_{t-h}\right) .
\end{aligned}
$$


By the dominated convergence Theorem, it follows that

$$
\begin{aligned}
\operatorname{Cov}\left(\sqrt{n} J^{-1} Y_{n}, \sqrt{n} \operatorname{vec} \gamma(\ell)\right) & \rightarrow-\sum_{h=-\infty}^{+\infty} 2 J^{-1} \operatorname{Cov}\left(\frac{\partial e_{t}^{\prime}\left(\theta_{0}\right)}{\partial \theta} \Sigma_{e 0}^{-1} e_{t}, e_{t-h-\ell} \otimes e_{t-h}\right) \\
& =-\sum_{h=-\infty}^{+\infty} 2 J^{-1} E\left(\frac{\partial e_{t}^{\prime}\left(\theta_{0}\right)}{\partial \theta} \Sigma_{e 0}^{-1} e_{t}\left\{e_{t-\ell-h} \otimes e_{t-h}\right\}^{\prime}\right)
\end{aligned}
$$

Then we have

$$
\Sigma_{\gamma_{m}, \hat{\theta}_{n}}^{\prime}=-2 J^{-1} \sum_{h=-\infty}^{+\infty} E\left(\frac{\partial e_{t}^{\prime}\left(\theta_{0}\right)}{\partial \theta} \Sigma_{e 0}^{-1} e_{t}\left\{\left(\begin{array}{c}
e_{t-1-h} \\
\vdots \\
e_{t-m-h}
\end{array}\right) \otimes e_{t-h}\right\}\right)
$$

Applying the central limit Theorem (CLT) for mixing processes (see Herrndorf, 1984) we directly obtain

$$
\begin{aligned}
\lim _{n \rightarrow \infty} \operatorname{Var}\left(\sqrt{n} J^{-1} Y_{n}\right) & =J^{-1} I J^{-1} \\
& =\Sigma_{\hat{\theta}_{n}}
\end{aligned}
$$

which gives the asymptotic covariance matrix of Theorem 4.1. It is clear that the existence of these matrices is ensured by the Davydov (1968) inequality. The proof is then complete.

Proof of Theorem 5.1. Recall that

$$
e_{t}(\theta)=X_{t}-\sum_{i=1}^{\infty} C_{i}(\theta) X_{t-i}=\mathbf{B}_{\theta}^{-1}(L) \mathbf{A}_{\theta}(L) X_{t}
$$

where $\mathbf{A}_{\theta}(L)=I_{d}-\sum_{i=1}^{p} \mathbf{A}_{i} L^{i} \quad$ and $\quad \mathbf{B}_{\theta}(L)=I_{d}-\sum_{i=1}^{q} \mathbf{B}_{i} L^{i}$ with $\mathbf{A}_{i}=$ $A_{0}^{-1} A_{i}$ and $\mathbf{B}_{i}=A_{0}^{-1} B_{i} B_{0}^{-1} A_{0}$. For $\ell=1, \ldots, p$ and $\ell^{\prime}=1, \ldots, q$, let $\mathbf{A}_{\ell}=\left(a_{i j, \ell}\right)$ and $\mathbf{B}_{\ell^{\prime}}=\left(b_{i j, \ell^{\prime}}\right)$. We define the matrices $\mathbf{A}_{i j, h}^{*}$ and $\mathbf{B}_{i j, h}^{*}$ by

$\mathbf{B}_{\theta}^{-1}(z) E_{i j}=\sum_{h=0}^{\infty} \mathbf{A}_{i j, h}^{*} z^{h}, \quad \mathbf{B}_{\theta}^{-1}(z) E_{i j} \mathbf{B}_{\theta}^{-1}(z) \mathbf{A}_{\theta}(z)=\sum_{h=0}^{\infty} \mathbf{B}_{i j, h}^{*} z^{h}, \quad|z| \leq 1$

for $h \geq 0$, where $E_{i j}=\partial \mathbf{A}_{\ell} / \partial a_{i j, \ell}=\partial \mathbf{B}_{\ell^{\prime}} / \partial b_{i j, \ell^{\prime}}$ is the $d \times d$ matrix with 1 at position $(i, j)$ and 0 elsewhere. Take $\mathbf{A}_{i j, h}^{*}=\mathbf{B}_{i j, h}^{*}=0$ when $h<0$. For 
any $a_{i j, \ell}$ and $b_{i j, \ell^{\prime}}$, we respectively write the multivariate residual derivatives

$$
\frac{\partial e_{t}}{\partial a_{i j, \ell}}=-\mathbf{B}_{\theta}^{-1}(L) E_{i j} X_{t-\ell}=-\sum_{h=0}^{\infty} \mathbf{A}_{i j, h}^{*} X_{t-h-\ell}
$$

and

$$
\frac{\partial e_{t}}{\partial b_{i j, \ell^{\prime}}}=\mathbf{B}_{\theta}^{-1}(L) E_{i j} \mathbf{B}_{\theta}^{-1}(L) \mathbf{A}_{\theta}(L) X_{t-\ell^{\prime}}=\sum_{h=0}^{\infty} \mathbf{B}_{i j, h}^{*} X_{t-h-\ell^{\prime}}
$$

On the other hand, considering $\hat{\Gamma}(h)$ and $\gamma(h)$ as values of the same function at the points $\hat{\theta}_{n}$ and $\theta_{0}$, a Taylor expansion about $\theta_{0}$ gives

$$
\begin{aligned}
\operatorname{vec} \hat{\Gamma}_{e}(h)=\operatorname{vec} \gamma(h)+ & \frac{1}{n} \sum_{t=h+1}^{n}\left\{e_{t-h}(\theta) \otimes \frac{\partial e_{t}(\theta)}{\partial \theta^{\prime}}\right. \\
& \left.+\frac{\partial e_{t-h}(\theta)}{\partial \theta^{\prime}} \otimes e_{t}(\theta)\right\}_{\theta=\theta_{n}^{*}}\left(\hat{\theta}_{n}-\theta_{0}\right)+O_{P}(1 / n) \\
= & \operatorname{vec} \gamma(h)+E\left(e_{t-h}\left(\theta_{0}\right) \otimes \frac{\partial e_{t}\left(\theta_{0}\right)}{\partial \theta^{\prime}}\right)\left(\hat{\theta}_{n}-\theta_{0}\right)+O_{P}(1 / n),
\end{aligned}
$$

where $\theta_{n}^{*}$ is between $\hat{\theta}_{n}$ and $\theta_{0}$. The last equality follows from the consistency of $\hat{\theta}_{n}$ and the fact that $\left(\partial e_{t-h} / \partial \theta^{\prime}\right)\left(\theta_{0}\right)$ is not correlated with $e_{t}$ when $h \geq 0$. Then for $h=1, \ldots, m$,

$$
\hat{\Gamma}_{m}:=\left(\left\{\operatorname{vec} \hat{\Gamma}_{e}(1)\right\}^{\prime}, \ldots,\left\{\operatorname{vec} \hat{\Gamma}_{e}(m)\right\}^{\prime}\right)^{\prime}=\gamma_{m}+\Phi_{m}\left(\hat{\theta}_{n}-\theta_{0}\right)+O_{P}(1 / n),
$$

where

$$
\Phi_{m}=E\left\{\left(\begin{array}{c}
e_{t-1} \\
\vdots \\
e_{t-m}
\end{array}\right) \otimes \frac{\partial e_{t}\left(\theta_{0}\right)}{\partial \theta^{\prime}}\right\} .
$$

In $\Phi_{m}$, one can express $\left(\partial e_{t} / \partial \theta^{\prime}\right)\left(\theta_{0}\right)$ in terms of the multivariate derivatives (16) and (17). From Theorem 4.1, we have obtained the asymptotic joint distribution of $\gamma_{m}$ and $\hat{\theta}_{n}-\theta_{0}$, which shows that the asymptotic distribution of $\sqrt{n} \hat{\Gamma}_{m}$, is normal, with mean zero and covariance matrix

$$
\begin{aligned}
\lim _{n \rightarrow \infty} \operatorname{Var}\left(\sqrt{n} \hat{\Gamma}_{m}\right)= & \lim _{n \rightarrow \infty} \operatorname{Var}\left(\sqrt{n} \gamma_{m}\right)+\Phi_{m} \lim _{n \rightarrow \infty} \operatorname{Var}\left(\sqrt{n}\left(\hat{\theta}_{n}-\theta_{0}\right)\right) \Phi_{m}^{\prime} \\
& +\Phi_{m} \lim _{n \rightarrow \infty} \operatorname{Cov}\left(\sqrt{n}\left(\hat{\theta}_{n}-\theta_{0}\right), \sqrt{n} \gamma_{m}\right) \\
& +\lim _{n \rightarrow \infty} \operatorname{Cov}\left(\sqrt{n} \gamma_{m}, \sqrt{n}\left(\hat{\theta}_{n}-\theta_{0}\right)\right) \Phi_{m}^{\prime} \\
= & \Sigma_{\gamma_{m}}+\Phi_{m} \Sigma_{\hat{\theta}_{n}} \Phi_{m}^{\prime}+\Phi_{m} \Sigma_{\hat{\theta}_{n}, \gamma_{m}}+\Sigma_{\hat{\theta}_{n}, \gamma_{m}} \Phi_{m}^{\prime} .
\end{aligned}
$$


From a Taylor expansion about $\theta_{0}$ of vec $\hat{\Gamma}_{e}(0)$ we have, vec $\hat{\Gamma}_{e}(0)=\operatorname{vec} \gamma(0)+$ $O_{P}\left(n^{-1 / 2}\right)$. Moreover, $\sqrt{n}(\operatorname{vec} \gamma(0)-E \operatorname{vec} \gamma(0))=O_{P}(1)$ by the CLT for mixing processes. Thus $\sqrt{n}\left(\hat{S}_{e} \otimes \hat{S}_{e}-S_{e} \otimes S_{e}\right)=O_{P}(1)$ and, using (5) and the ergodic Theorem, we obtain

$$
\begin{aligned}
& n\left\{\operatorname{vec}\left(\hat{S}_{e}^{-1} \hat{\Gamma}_{e}(h) \hat{S}_{e}^{-1}\right)-\operatorname{vec}\left(S_{e}^{-1} \hat{\Gamma}_{e}(h) S_{e}^{-1}\right)\right\} \\
= & n\left\{\left(\hat{S}_{e}^{-1} \otimes \hat{S}_{e}^{-1}\right) \operatorname{vec} \hat{\Gamma}_{e}(h)-\left(S_{e}^{-1} \otimes S_{e}^{-1}\right) \operatorname{vec} \hat{\Gamma}_{e}(h)\right\} \\
= & n\left\{\left(\hat{S}_{e} \otimes \hat{S}_{e}\right)^{-1} \operatorname{vec} \hat{\Gamma}_{e}(h)-\left(S_{e} \otimes S_{e}\right)^{-1} \operatorname{vec} \hat{\Gamma}_{e}(h)\right\} \\
= & \left(\hat{S}_{e} \otimes \hat{S}_{e}\right)^{-1} \sqrt{n}\left(S_{e} \otimes S_{e}-\hat{S}_{e} \otimes \hat{S}_{e}\right)\left(S_{e} \otimes S_{e}\right)^{-1} \sqrt{n} \operatorname{vec} \hat{\Gamma}_{e}(h) \\
= & O_{P}(1) .
\end{aligned}
$$

In the previous equalities, we also use $\operatorname{vec}(A B C)=\left(C^{\prime} \otimes A\right) \operatorname{vec}(B)$ and $(A \otimes B)^{-1}=A^{-1} \otimes B^{-1}$ when $\mathrm{A}$ and $\mathrm{B}$ are invertible. It follows that

$$
\begin{aligned}
\hat{\rho}_{m} & =\left(\left\{\operatorname{vec} \hat{R}_{e}(1)\right\}^{\prime}, \ldots,\left\{\operatorname{vec} \hat{R}_{e}(m)\right\}^{\prime}\right)^{\prime} \\
& =\left(\left\{\left(\hat{S}_{e} \otimes \hat{S}_{e}\right)^{-1} \operatorname{vec} \hat{\Gamma}_{e}(1)\right\}^{\prime}, \ldots,\left\{\left(\hat{S}_{e} \otimes \hat{S}_{e}\right)^{-1} \operatorname{vec} \hat{\Gamma}_{e}(m)\right\}^{\prime}\right)^{\prime} \\
& =\left\{I_{m} \otimes\left(\hat{S}_{e} \otimes \hat{S}_{e}\right)^{-1}\right\} \hat{\Gamma}_{m}=\left\{I_{m} \otimes\left(S_{e} \otimes S_{e}\right)^{-1}\right\} \hat{\Gamma}_{m}+O_{P}\left(n^{-1}\right)
\end{aligned}
$$

We now obtain (6) from (5). Hence, we have

$$
\operatorname{Var}\left(\sqrt{n} \hat{\rho}_{m}\right)=\left\{I_{m} \otimes\left(S_{e} \otimes S_{e}\right)^{-1}\right\} \Sigma_{\hat{\Gamma}_{m}}\left\{I_{m} \otimes\left(S_{e} \otimes S_{e}\right)^{-1}\right\} .
$$

This completes the proof.

Proof of Theorem 6.2. The proof is similar to that given by Francq, Roy and Zakoïan (2003) for Theorem 5.2.

\section{Acknowledgements}

The author gratefully acknowledges the quick and careful reading of the manuscript by the two anonymous referees. Their detailed comments led to greatly improve the presentation. 
[1] Ahn, S. K. (1988) Distribution for residual autocovariances in multivariate autoregressive models with structured parameterization. Biometrika $75,590-93$.

[2] Andrews, D. W. K. (1991) Heteroskedasticity and autocorrelation consistent covariance matrix estimation. Econometrica 59, 817-858.

[3] Arbués, I. (2008) An extended portmanteau test for VARMA models with mixing nonlinear constraints, Journal of Time Series Analysis 29, $741-761$.

[4] Berk, K. N. (1974) Consistent Autoregressive Spectral Estimates. Annals of Statistics 2, 489-502.

[5] Bauwens, L., Laurent, S. and Rombouts, J. V. K. (2006) Multivariate GARCH models: a survey. Journal of Applied Econometrics 21, 79-109.

[6] Boubacar Mainassara, Y. (2010) Selection of weak VARMA models by modified Akaike's information criteria. Working Papers, http://mpra.ub.uni-muenchen.de/24981/.

[7] Boubacar Mainassara, Y. and Francq, C. (2010) Estimating structural VARMA models with uncorrelated but non-independent error terms. Journal of Multivariate Analysis, to appear.

[8] Box, G. E. P. and Pierce, D. A. (1970) Distribution of residual autocorrelations in autoregressive integrated moving average time series models. Journal of the American Statistical Association 65, 1509-26.

[9] Brockwell, P. J. and Davis, R. A. (1991) Time series: theory and methods. Springer Verlag, New York.

[10] Chabot-Hallé, D. and Duchesne, P. (2008) Diagnostic checking of multivariate nonlinear time series models with martingale difference errors, Statistics and Probability Letters 78, 997-1005.

[11] Chitturi, R. V. (1974) Distribution of residual autocorrelations in multiple autoregressive schemes. Journal of the American Statistical Association 69, 928-934.

[12] Chitturi, R. V. (1976) Distribution of multivariate white noise autocorrelations. Journal of the American Statistical Association 71, 223-226. 
[13] Davydov, Y. A. (1968) Convergence of Distributions Generated by Stationary Stochastic Processes. Theory of Probability and Applications 13, 691-696.

[14] den Hann, W. J. and Levin, A. (1997) A Practitioner's Guide to Robust Covariance Matrix Estimation. In Handbook of Statistics 15, Rao, C.R. and G.S. Maddala (eds), 291-341.

[15] Dufour, J-M. and Pelletier, D. (2005) Practical methods for modelling weak VARMA processes: identification, estimation and specification with a macroeconomic application. Technical report, Département de sciences économiques and CIREQ, Université de Montréal, Montréal, Canada.

[16] Francq, C. and Raïssi, H. (2007) Multivariate Portmanteau Test for Autoregressive Models with Uncorrelated but Nonindependent Errors, Journal of Time Series Analysis 28, 454-470.

[17] Francq, C., Roy, R. and Zakoïan, J-M. (2003) Goodness-of-fit tests for ARMA models with uncorrelated errors, Discussion Paper CRM2925, Centre de recherches mathématiques, Université de Montréal. http://www.crm.umontreal.ca/pub/Rapports/2900-2999/2925.pdf

[18] Francq, C., Roy, R. and Zakoïan, J-M. (2005) Diagnostic checking in ARMA Models with Uncorrelated Errors, Journal of the American Statistical Association 100, 532-544.

[19] Francq, C. and Zakoïan, J-M. (1998) Estimating linear representations of nonlinear processes, Journal of Statistical Planning and Inference 68, $145-165$.

[20] Francq, C. and Zakoïan, J-M. (2005) Recent results for linear time series models with non independent innovations. In Statistical Modeling and Analysis for Complex Data Problems, Chap. 12 (eds P. DuCHesne and B. RÉmillard). New York: Springer Verlag, 241-265.

[21] Hannan, E. J. (1976) The identification and parametrization of ARMAX and state space forms, Econometrica 44, 713-723. 
[22] Herrndorf, N. (1984) A Functional Central Limit Theorem for Weakly Dependent Sequences of Random Variables. The Annals of Probability $12,141-153$.

[23] Hong, Y. (1996) Consistent testing for serial correlation of unknown form, Econometrica 64, 837-864.

[24] Hosking, J. R. M. (1980) The multivariate portmanteau statistic, Journal of the American Statistical Association 75, 602-608.

[25] Hosking, J. R. M. (1981a) Equivalent forms of the multivariate portmanteau statistic, Journal of the Royal Statistical Society B 43, 261-262.

[26] Hosking, J. R. M. (1981b) Lagrange-tests of multivariate time series models, Journal of the Royal Statistical Society B 43, 219-230.

[27] Imhof, J. P.] (1961) Computing the distribution of quadratic forms in normal variables. Biometrika 48, 419-426.

[28] Jeantheau, T. (1998) Strong consistency of estimators for multivariate ARCH models, Econometric Theory 14, 70-86.

[29] Li, W. K. and McLeod, A. I. (1981) Distribution of the residual autocorrelations in multivariate ARMA time series models, Journal of the Royal Statistical Society B 43, 231-239.

[30] Ljung, G. M. and Box, G. E. P. (1978) On measure of lack of fit in time series models. Biometrika 65, 297-303.

[31] Lütkepohl, H.] (2005) New introduction to multiple time series analysis. Springer Verlag, Berlin.

[32] Newey, W. K. and West, K. D. (1987) A simple, positive semi-definite, heteroskedasticity and autocorrelation consistent covariance matrix. Econometrica 55, 703-708.

[33] Reinsel, G. C. (1997) Elements of multivariate time series Analysis. Second edition. Springer Verlag, New York.

[34] Romano, J. L. and Thombs, L. A. (1996) Inference for autocorrelations under weak assumptions, Journal of the American Statistical Association 91, 590-600. 